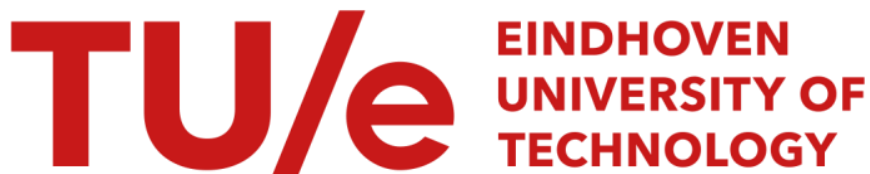

\section{Successive-capture analysis of RTS/CTS in ad-hoc networks}

Citation for published version (APA):

Ho, C. K., \& Linnartz, J. P. M. G. (2008). Successive-capture analysis of RTS/CTS in ad-hoc networks. IEEE

Transactions on Wireless Communications, 7(1), 213-223. https://doi.org/10.1109/TWC.2008.060454

DOI:

10.1109/TWC.2008.060454

Document status and date:

Published: 01/01/2008

\section{Document Version:}

Publisher's PDF, also known as Version of Record (includes final page, issue and volume numbers)

\section{Please check the document version of this publication:}

- A submitted manuscript is the version of the article upon submission and before peer-review. There can be important differences between the submitted version and the official published version of record. People interested in the research are advised to contact the author for the final version of the publication, or visit the $\mathrm{DOI}$ to the publisher's website.

- The final author version and the galley proof are versions of the publication after peer review.

- The final published version features the final layout of the paper including the volume, issue and page numbers.

Link to publication

\section{General rights}

Copyright and moral rights for the publications made accessible in the public portal are retained by the authors and/or other copyright owners and it is a condition of accessing publications that users recognise and abide by the legal requirements associated with these rights.

- Users may download and print one copy of any publication from the public portal for the purpose of private study or research.

- You may not further distribute the material or use it for any profit-making activity or commercial gain

- You may freely distribute the URL identifying the publication in the public portal.

If the publication is distributed under the terms of Article $25 f a$ of the Dutch Copyright Act, indicated by the "Taverne" license above, please follow below link for the End User Agreement:

www.tue.nl/taverne

Take down policy

If you believe that this document breaches copyright please contact us at:

openaccess@tue.nl

providing details and we will investigate your claim. 


\title{
Successive-Capture Analysis of RTS/CTS in Ad-Hoc Networks
}

\author{
Chin Keong Ho, Member, IEEE, and Jean-Paul M. G. Linnartz, Senior Member, IEEE
}

\begin{abstract}
We consider a wireless ad-hoc network with random ALOHA transmissions between nodes. However nodes can decide to use the Request-to-Send (RTS) / Clear-to-Send (CTS) protocol for occasional long packets. Via the RTS/CTS protocol, nodes proactively make a temporal and spatial reservation of the channel before sending the actual data payload (DATA). The aim of this paper is to explore the effectiveness of the RTS/CTS protocol in such a wireless network, in particular by alleviating the commonly made assumption that an entire RTS/CTS/DATA cycle is always successful if only the RTS packet is recovered. Our results show that this assumption loses its accuracy if the data rate is optimized for the link throughput or if one aims for allowing dense frequency reuse. We quantify the spatial and temporal impact of the RTS/CTS reservation on the network traffic, as well as the link throughput achieved. Numerical results demonstrate that for sufficiently long packets, a significant throughput gain can be achieved by employing the RTS/CTS reservation.
\end{abstract}

Index Terms-Medium access control, RTS/CTS, capture effect, throughput maximization, rate adaptation, ALOHA.

\section{INTRODUCTION}

A D-HOC networks can provide wireless connectivity even in the absence of a fixed network infrastructure [1]. In such networks, distributed peer-to-peer communications are carried out typically over a common frequency band. Fig. 1(a) shows an ad-hoc network with source-destination pairs $\mathrm{S} \rightarrow$ $\mathrm{D}$ and $\mathrm{S}_{i} \rightarrow \mathrm{D}_{i}$. Each source node is shown to transmit a message to its destination node directly, but it is possible also to transmit via multiple hops using other intermediate nodes as relays.

In an infrastructure-based wireless network, on the other hand, an access point (AP) acts as the gateway to the infrastructure. Mutual interference is reduced by appropriately assigning different frequencies to neighboring APs. This assignment, however, is usually performed in an ad-hoc fashion in the home wireless LANs, as compared to the centralized planning used in mobile telephony. Wireless nodes, known as stations (STAs), send or receive data payload (DATA) packets

Manuscript received July 8, 2006; revised April 3, 2007 and August 29, 2007; accepted September 3, 2007. The associate editor coordinating the review of this paper and approving it for publication was M. Zorzi. This paper was presented in part at the 27th Symposium on Information Theory in the Benelux, Noordwijk, The Netherlands, June 2006, and 17th Annual IEEE Symposium on PIMRC, Helsinki, Finland, September 2006.

C. K. Ho is with the Institute for Infocomm Research, 21 Heng Mui Keng Terrace, 119613 Singapore. He conducted this work while with the Department of Electrical Engineering, Eindhoven University of Technology, The Netherlands, and with Philips Research Laboratories, Eindhoven, The Netherlands (e-mail: hock@i2r.a-star.edu.sg).

J. P. M. G. Linnartz is with Philips Research Laboratories, Eindhoven, The Netherlands, and with the Department of Electrical Engineering, Eindhoven University of Technology, The Netherlands (e-mail: j.p.linnartz@philips.com). Digital Object Identifier 10.1109/TWC.2008.060454.

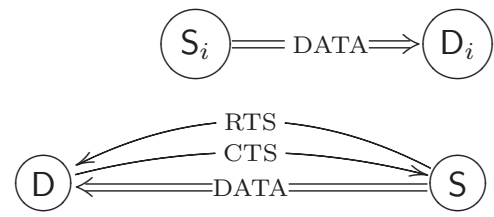

(a) Ad-hoc network with peer-to-peer transmissions, where sources $S, S_{i}$ send DATA to $\mathrm{D}, \mathrm{D}_{i}$, respectively.

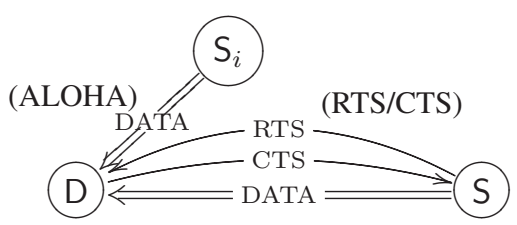

(b) Infrastructure network with uplink transmissions, where sources $S, S_{i}$ (the STAs) send DATA to a common destination D (the AP).

Fig. 1. Using ALOHA and RTS/CTS in different networks.

via the AP in a cell. In a downlink, the STA is the destination, while the AP is the source. In an uplink, their roles are reversed, see Fig. 1(b). Here, two STAs depicted as sources $S_{i}$ and $S$ compete to send their DATA to the AP, their common destination $\mathrm{D}$.

\section{A. MAC Protocols}

1) Infrastructure Networks: To support a variable number of active nodes, a random access MAC protocol is employed. Well-established random access protocols for the infrastructure networks include ALOHA [2], physical carrier sensing [3] and virtual carrier sensing - also known as the Requestto-Send/Clear-to-Send (RTS/CTS) protocol [4], [5]. In the ALOHA protocol, a node transmits whenever new data arrives, or when this needs to be retransmitted because of a missing acknowledgement (ACK). In the slotted ALOHA variant, transmissions are also globally synchronized. Although simple to implement, ALOHA suffers from excessive interference due to conflicting transmissions when the network load is high [6]. Collisions can be mitigated by physical carrier sensing, where a node is inhibited to transmit if, prior to transmission, it detects signal power from ongoing transmissions. However, physical carrier sensing suffers from the well-known hiddennode problem. The RTS/CTS protocol can partly solve this problem.

The RTS/CTS protocol was first proposed as the MACA protocol [4] where physical carrier sensing is replaced by virtual carrier sensing: the source first informs the destination of its intention to exchange data by issuing an RTS packet, and the destination confirms this with a CTS packet, after 


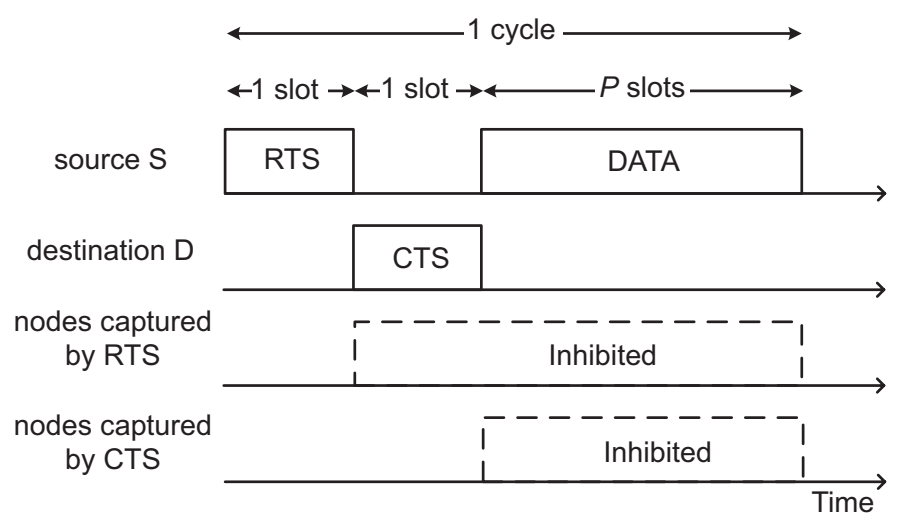

Fig. 2. An idealized RTS/CTS protocol. An ACK can be additionally sent to inform the destination that DATA is recovered.

which the source sends the DATA packet. All other nodes (including hidden nodes) that recover the RTS or CTS packet are inhibited from transmitting during some specified time interval, to facilitate a successful RTS-CTS-DATA cycle. This RTS/CTS cycle can be extended by an ACK packet from the destination to reduce the delay caused by erroneous cycles at the transport layer [5]. In Fig. 1(b), the ALOHA protocol is used by $S_{i}$ to send data to $D$. To protect longer packets, the RTS/CTS protocol is used by $\mathrm{S}$ to send data to D.

Current IEEE 802.11 [7] systems are mostly set up as infrastructure networks, where the RTS/CTS protocol is implemented in the distributed co-ordination function (DCF). An idealized timing model of the protocol is given in Fig. 2. An ACK phase can be additionally appended to confirm that data has been recovered.

In an infrastructure network, the STAs only communicate with the AP, so only a single transmission can be successful at any time. Spatial reuse is not required within each cell. Consequently, the lowest possible rates are used to encode the RTS and CTS packets so that the inhibition practically covers the entire cell area. Further, physical carrier sensing is always used concurrently with RTS/CTS in IEEE 802.11.

2) Ad-hoc Networks: In an ad-hoc network, however, multiple simultaneous but spatially separated transmissions are possible, so having extensive inhibition increases the number of exposed nodes, limits spatial reuse and can lead to dead locks [8]. As such, the IEEE 802.11 DCF is not suitable for an ad-hoc network, see for example [8], [9]. Preferably, other transmissions should be allowed as long as they do not interfere harmfully with the RTS/CTS cycle. Compared to an infrastructure network with interference coming only within a cell, there is more emphasis on a well-designed MAC protocol in an ad-hoc network, and its spatial demarcation of reservation areas becomes an interesting object of study.

\section{B. Successive-Capture Analysis}

Analysis of a simplified or approximate RTS/CTS model provides insights that complement simulation results, such as in [8], [9]. In the pioneering analysis in [10], a packet was assumed to be recovered if and only if no other concurrent transmission occurs. This model can be improved by considering capture, depending on path losses and fading of all links, including interference propagation paths. For this purpose, it is common to assume that the locations of interferers follow a homogeneous Poisson process [6], [11]-[15]. It can also be justified for ad-hoc communications, particularly if nodes belong to separate groups, or in spatially overlapping infrastructure-based networks operated by different owners.

In one capture model, perfect reception of a packet transmitted from a certain distance is assumed if no other interfering packet transmission occurred within some fraction of that distance [11]. An improved packet detection model is based on the capture effect whereby packets are considered to be recovered (even in the presence of interfering signals) if and only if the signal-to-noise plus interference ratio (SINR) is larger than a certain capture ratio [6], [12]-[15]. The RTS/CTS protocol has also been analyzed considering the capture effect under Rayleigh fading in [16], and under both Rayleigh and shadow fading in [17].

So far in literature [10], [16], [17], the RTS/CTS protocol has been modeled by assuming that if the RTS packet is recovered by the destination, then the CTS, DATA and ACK packets are always recovered too. This becomes optimistic for environments with dense (or even contiguous) spatial reuse. The boundaries of the inhibited area are fuzzy and harmful transmission from these fringes are likely. Our model considers more realistic, statistically dependent capture probabilities as the protocol progresses; we call this the successive-capture analysis. In particular, it is possible for packets in later phases of the protocol to fail even if the earlier phases have succeeded.

\section{Scenario}

In a wireless ad-hoc network, there are many applications in which small amounts of sensor data are sent frequently, while a larger amount of consolidated data is sent occasionally, usually after data aggregation and fusion. An example is in a hospital where routine vital signs of patients are uploaded wirelessly to a database, while the full medical record of a patient is downloaded by a doctor occasionally, particularly in situations requiring medical attention. The short, routine messages can be sent efficiently using the ALOHA protocol, while the large amount of record data that requires higher protection can be protected by the RTS/CTS protocol.

In this paper, we consider an ad-hoc network where (long) packets can be protected by RTS/CTS if the sources deem this advantageous, while for the majority of (short) messages no reservation is made. All nodes listen and obey the inhibitions imposed by the RTS/CTS protocol. Our results also become relevant in infrastructure networks if the density of cells increase and many network administrators each assign frequency channels without mutual coordination.

\section{Contributions}

The objective of this paper is to understand, analyze and optimize the RTS/CTS protocol. The effects of the wireless channels are taken into account by employing the successivecapture analysis. We derive a probabilistic description of the time-varying inhibition area from which a reduced amount of interfering traffic arrives as the RTS/CTS protocol progresses. These descriptions lead to capture probabilities in successive 
phases of the protocol, and eventually to the achieved link throughput. We confirm (and quantify) that for moderately large messages, nodes can achieve a significantly higher throughput when using the RTS/CTS protocol, compared to the ALOHA protocol. From our investigations we further conclude that for given RTS and CTS rates, there is an optimum rate for the DATA packets. Such data rate optimization cannot be obtained without the use of successivecapture analysis, since conventional capture analysis does not adequately account for the effects of data rate on the performance of the RTS/CTS protocol.

The paper is organized as follows. Section II specifies the system model. Section III derives the throughput achieved using the RTS/CTS protocol. Then, Section IV derives the conditional capture probability in each phase of the RTS/CTS cycle. Numerical results are given in Section V. Finally, Section VI concludes the paper.

\section{MODEL}

We consider a two-dimensional wireless ad-hoc network operating in a region $\mathcal{A}$, see Fig. 1(a). We classify nodes according to their roles as sources or destinations, but in a RTS/CTS cycle the participating source and destination alternatingly acts as a transmitter or a receiver. The $x, y$-coordinates of the tagged source $S$ and tagged destination $D$ are denoted by position vectors $\boldsymbol{a}_{\mathrm{S}}$ and $\boldsymbol{a}_{\mathrm{D}}$, respectively. Other transmitting sources $\mathrm{S}_{i}, i=1, \cdots, N$, are called interferers.

The network is dominated by short transmissions without RTS/CTS, while occasional long transmissions are protected by RTS/CTS. So we may reasonably assume that the RTS/CTS cycles do not overlap with each other, but nodes see interference from randomly arriving messages. Thus we focus on a single RTS/CTS cycle between S to D.

\section{A. RTS/CTS Protocol}

The source $S$ uses the RTS/CTS protocol to send DATA to the destination D, as summarized in Fig. 2. The RTS and CTS packets each uses one slot while the DATA packet uses $P$ slots. All (potential) interferers obey the inhibitions provided that they recover the RTS, CTS messages. Notations associated with the respective RTS, CTS and DATA phases are denoted with subscripts R, C, D. Time is slotted and synchronized.

The ACK is omitted to clarify our analytical approach and this allows direct benchmarking with the classical slotted ALOHA [2]. To isolate the effects of virtual carrier sensing, physical carrier sensing is disabled. This approach, also used in the MACA protocol [4], allows a higher spatial reuse to be realized in an ad-hoc network. Our analysis can nevertheless be extended when ACK is included or when physical carrier sensing is used.

If a packet is successfully recovered by the destination, we say that the packet captures the destination. Let $\mathcal{C}_{R}$ denote the event that a RTS slot captures $\mathrm{D}, \mathcal{C}_{\mathrm{C}}$ the event that a CTS slot captures $S$ and $\mathcal{C}_{\mathrm{D}}$ the event that a DATA slot captures D. We note that $\mathcal{C}_{\mathrm{R}}\left(\mathcal{C}_{\mathrm{C}}\right)$ implies that the RTS (CTS, respectively) packet is also recovered, while $\mathcal{C}_{\mathrm{D}}$ may not imply that the DATA packet, which may consist of multiple slots, is entirely recovered. Since capture events have a sequential and causal relation,

$$
\begin{aligned}
\mathcal{C}_{\mathrm{C}} & \Leftrightarrow \mathcal{C}_{\mathrm{R}} \cap \mathcal{C}_{\mathrm{C}} ; \\
\mathcal{C}_{\mathrm{D}} & \Leftrightarrow \mathcal{C}_{\mathrm{R}} \cap \mathcal{C}_{\mathrm{C}} \cap \mathcal{C}_{\mathrm{D}} .
\end{aligned}
$$

We only spell out the full sequences of events in expressions for conditional probabilities when this contributes to the intuition; otherwise we only denote the last event in time. The intersection notation $\cap$ is replaced with a comma for brevity when it is obvious.

\section{B. Wireless Network Model}

1) Traffic: The traffic in a slot is fully described by the number and positions of active interferers within an operation region $\mathcal{A}$ :

$$
\mathbb{T}=\left\{\mathcal{N},\left\{\boldsymbol{a}_{i} \in \mathcal{A}\right\}_{i=1}^{\mathcal{N}}\right\}
$$

The density of the traffic, written as $f_{\mathbb{T}}\left(\mathcal{N},\left\{\boldsymbol{a}_{i}\right\}_{i=1}^{\mathcal{N}}\right)=$ $\operatorname{Pr}(\mathcal{N}) \Pi_{i} f_{\boldsymbol{a}_{i}}\left(\boldsymbol{a}_{i} \mid \mathcal{N}\right)$, consists of products of a probability mass function (pmf) and of probability density functions (pdfs). We consider the limiting case where $\mathcal{A}$ grows infinitely large and $\mathcal{N} \rightarrow \infty$. Therefore, nonzero throughput is possible only because of the capture effect and a sufficiently steep path loss law.

In the RTS slot, the traffic is denoted as $\mathbb{T}_{\mathrm{R}}$. In the CTS slot, the traffic is denoted as $\mathbb{T}_{\mathrm{C}}$. The DATA phase spans $P$ slots and the traffic for slot $k$ is denoted as $\mathbb{T}_{\mathrm{D}}(k), k=1, \cdots, P$. Finally, the cycle traffic, $\mathbb{T}_{\text {cycle }} \triangleq$ $\left\{\mathbb{T}_{\mathrm{R}}, \mathbb{T}_{\mathrm{C}}, \mathbb{T}_{\mathrm{D}}(1), \cdots, \mathbb{T}_{\mathrm{D}}(P)\right\}$, describes the traffic of all the slots in an RTS/CTS cycle. Due to causality (1), the CTS traffic $\mathbb{T}_{\mathrm{C}}$ is defined only if the RTS is recovered, and the DATA traffic $\mathbb{T}_{\mathrm{D}}$ is defined only if the RTS and CTS are recovered.

We assume that DATA transmissions are independent, i.e., we ignore possible correlation due to retransmissions [6] or deferred transmissions. This allows us to study the traffic resulting directly from the RTS/CTS inhibitions. For the RTS slot (and also for the ALOHA protocol), for reasons argued before, we describe the traffic as a Poisson process with traffic intensity $\mathcal{G}(\boldsymbol{a})$ packets per time slot per unit area (ppsa), where $a \in \mathcal{A}$. Specifically, we assume a homogeneous Poisson process with $\mathcal{G}(\boldsymbol{a})=G_{o}$. For the other slots later in the RTS/CTS cycle, we shall argue in Section IV that the traffic is well described by non-homogeneous Poisson processes.

Generally, for a Poisson process, the density of traffic $\mathbb{T}$ is determined by the traffic intensity $\mathcal{G}(\boldsymbol{a})$, as follows. Within $\mathcal{A}, \mathcal{N}$ is Poisson distributed with an expected value of $\widetilde{G}=\int_{\boldsymbol{a} \in \mathcal{A}} \mathcal{G}(\boldsymbol{a}) \mathrm{d} \boldsymbol{a}$ packets per time slot (pps). Given $\mathcal{N}$, the locations of the interferers are i.i.d with each pdf given by $f_{\boldsymbol{a}}(\boldsymbol{a} \mid \mathcal{N})=\mathcal{G}(\boldsymbol{a}) / \widetilde{G}, \boldsymbol{a} \in \mathcal{A}$.

2) Path Loss: All transmitters use the same power. However, each signal experiences a path loss depending on the propagation distance, $a$. The local-mean power, i.e., the averaged received power for a given $a$, follows the path loss law $\bar{\gamma}=a^{-\beta}$. We use a path loss exponent of $\beta=4$. 
3) Wireless Channel: We assume that all transmitterreceiver links experience flat Rayleigh fading, so the power $\gamma$ received over a distance $a$ follows an exponential distribution around the local-mean power $\bar{\gamma}$, i.e., $f_{\gamma}(\gamma)=\exp (-\gamma / \bar{\gamma}) / \bar{\gamma}$. We assume that all channels are quasi-static at least over one slot. The channel between $S$ and $D$, however, may vary during the RTS/CTS cycle:

- Quasi-Static (QS) channel: the SD channel is the same for all slots;

- Quasi-static, non-reciprocal (QSNR) channel: the forward channel from $S$ to D (in the RTS and DATA phases) is independent of the reverse channel from $D$ to $S$ (in the CTS and ACK phases), but both channels are constant;

- Independent and identically distributed (I.I.D.) channel: the SD channel is i.i.d. for all slots.

The QS channel represents a reciprocal channel (i.e., the forward and reverse channels are the same) with a fixed channel fading throughout the RTS/CTS cycle. The QSNR channel reflects a non-reciprocal channel with independent forward and reverse channels, but still quasi-static. The I.I.D. channel is sometimes called the block fading channel in literature [18]. The results obtained for this channel are also relevant for a slow, correlated fading when $P$ is sufficiently large, since it leads to a diverse collection of SINRs in the DATA phase.

\section{Capture Model}

The instantaneous SINR is defined as

$$
\operatorname{SINR}=\frac{\gamma^{\text {sig }}}{\gamma^{\text {int }}}, \quad \gamma^{\text {int }}=N_{\mathrm{o}}+\sum_{i=1}^{\mathcal{N}} \gamma_{i},
$$

where $\gamma^{\text {sig }}$ is the instantaneous power of the wanted signal, and $\gamma^{\text {int }}$ is the sum of the noise power $N_{\mathrm{o}}$ and the instantaneous power of the $i^{t h}$ interferer $\gamma_{i}$, where $i=1,2, \cdots, \mathcal{N}$. We treat the SINR as a random variable that depends on the random variables $\gamma^{\text {sig }}, \gamma^{\text {int }}$. When the SINR is larger than a fixed capture ratio, we assume that the packet captures the destination.

The mutual information between a transmitted packet and the received packet is $\mathcal{I} \triangleq \log _{2}(1+$ SINR $)$ using an ideal Gaussian code. According to information theoretic results for Gaussian channels [19], a packet encoded at data rate $R$ would experience an information outage if $\mathcal{I}<R$, or equivalently if

$$
\operatorname{SINR}<z(R) \triangleq 2^{R}-1 \text {. }
$$

Here, $z(R)$ is also the capture ratio. In practice, capture requires higher SINR, but we follow [20] by assuming that a capture occurs if and only if (3) is satisfied. This approximation predicts the capture probability accurately within a few $\mathrm{dBs}$ of SNR (or SINR in this case) using practical trellis codes [18]. Practical codes can thus be accounted for by adding a margin to $z(R)$.

\section{Capture Model in Different Phases of RTS/CTS Cycle}

Let $\gamma_{\Phi}^{\text {sig }}, \gamma_{\Phi}^{\text {int }}, R_{\Phi}$ and $z_{\Phi}=z\left(R_{\Phi}\right)$ denote, respectively, the signal power, total interference power, rate and capture ratio of a packet in phase $\Phi \in\{\mathrm{R}, \mathrm{C}, \mathrm{D}\}$. The RTS or CTS packet is one slot long. According to (3), for the RTS and CTS slots

$$
\mathcal{C}_{\Phi} \Leftrightarrow \frac{\gamma_{\Phi}^{\text {sig }}}{\gamma_{\Phi}^{\text {int }}}>z_{\Phi}, \Phi=\mathrm{R}, \mathrm{C} .
$$

Next, we consider two models for the capture of a given slot in the DATA phase.

1) Slot-by-Slot DATA Detection: In this mode, each slot in DATA is encoded independently, and hence also detected and recovered independently. We recall that the (ALOHA) interference at different slots is i.i.d. Hence, similar to (4), the DATA capture is determined as

$$
\mathcal{C}_{\mathrm{D}} \Leftrightarrow \frac{\gamma_{\mathrm{D}}^{\text {sig }}}{\gamma_{\mathrm{D}}^{\text {int }}}>z_{\mathrm{D}}
$$

In this approach, one bit is used to acknowledge each DATA slot, so multiple ACK bits are transmitted in the ACK packet. This approach is similar to the block-acknowledgement mode adopted in IEEE 802.11e as an optional feature to reduce the MAC overheads [21], where RTS/CTS is in fact recommended for protection.

2) Entire-Packet DATA Detection: Alternatively, all the data can be encoded as a (long) codeword and transmitted as a packet over $P$ slots. The DATA is thus detected as an entire packet. When the packet length is sufficiently large, an achievable rate in bit/symbol is given by the averaged mutual information $\overline{\mathcal{I}}_{\mathrm{D}} \triangleq \frac{1}{P} \sum \mathcal{I}_{\mathrm{D}, k}$ [20], where $\mathcal{I}_{\mathrm{D}, k}=$ $\log _{2}\left(1+\operatorname{SINR}_{k}\right)$ is the mutual information in slot $k$ of the DATA phase. Further, if a packet is recovered, every DATA slot in it must also be recovered. In accordance we model that

$$
\mathcal{C}_{\mathrm{D}} \Leftrightarrow \overline{\mathcal{I}}_{\mathrm{D}}>R_{\mathrm{D}}
$$

\section{SySteM PERFormanCE}

This section formulates the capture probability and link throughput for the RTS/CTS protocol. We fix $P$, rather than consider a composite traffic with packets of varying length, to investigate for which $P$ the RTS/CTS option becomes favorable.

\section{A. DATA Capture Probability}

From (1b), the DATA capture probability is

$$
\begin{aligned}
\operatorname{Pr}\left(\mathcal{C}_{\mathrm{D}}\right) & =\operatorname{Pr}\left(\mathcal{C}_{\mathrm{R}}, \mathcal{C}_{\mathrm{C}}, \mathcal{C}_{\mathrm{D}}\right) \\
& =\operatorname{Pr}\left(\mathcal{C}_{\mathrm{R}}\right) \operatorname{Pr}\left(\mathcal{C}_{\mathrm{C}} \mid \mathcal{C}_{\mathrm{R}}\right) \operatorname{Pr}\left(\mathcal{C}_{\mathrm{D}} \mid \mathcal{C}_{\mathrm{R}}, \mathcal{C}_{\mathrm{C}}\right) .
\end{aligned}
$$

We refine [10], [16], [17] by neither assuming that $\operatorname{Pr}\left(\mathcal{C}_{\mathrm{C}} \mid \mathcal{C}_{\mathrm{R}}\right)=1$ nor $\operatorname{Pr}\left(\mathcal{C}_{\mathrm{D}} \mid \mathcal{C}_{\mathrm{R}}, \mathcal{C}_{\mathrm{C}}\right)=1$.

\section{B. Throughput}

Since we assume that RTS/CTS cycles do not overlap, the cycle time, i.e., the duration of each RTS/CTS cycle, is i.i.d. Hence, the cycle time forms a renewal process. Let $s_{k} \in\left\{0, L_{\text {sym }} \times R_{\mathrm{D}}\right\}$ be the number of bits recovered in slot $k$, where $L_{\text {sym }}$ is the number of symbols sent per slot. We note that in the RTS and CTS phases $s_{k}=0$ since only overhead is 
sent. By applying the renewal-reward theorem [22], the time average of the throughput in bit/symbol is

$$
\begin{aligned}
\bar{s}\left(a_{s}, R_{\mathrm{R}}, R_{\mathrm{C}}, R_{\mathrm{D}}, P\right) & \triangleq \lim _{N \rightarrow \infty} \frac{1}{L_{\mathrm{sym}}} \frac{1}{N} \sum_{k=1}^{N} s_{k} \\
& =\frac{1}{L_{\mathrm{sym}}} \frac{\mathbb{E}[\mathcal{R}]}{\mathbb{E}[\mathcal{T}]}
\end{aligned}
$$

with probability one. Here, the expectation $\mathbb{E}$ is carried out over the events in one cycle; $\mathcal{R}$ is the reward in the form of the number of bits recovered in one cycle, and $\mathcal{T}$ is the cycle time in slots. The argument $a_{s} \triangleq\left|\boldsymbol{a}_{\mathrm{S}}-\boldsymbol{a}_{\mathrm{D}}\right|$ is defined as the distance between $\mathrm{S}$ and $\mathrm{D}$. As explicitly indicated above, $\bar{s}$ depends on many parameters, but we take $a_{s}, R_{\mathrm{R}}, R_{\mathrm{C}}$ to be fixed (and drop these arguments subsequently). We now analyze how the DATA rate $R_{\mathrm{D}}$ and DATA length $P$ affect the throughput.

Let us denote the complement of $\mathcal{C}$ as $\mathcal{E}$, i.e., $\mathcal{E}$ is the event that a slot is received in error. There are only two possible situations, S1 and S2, when a cycle terminates ${ }^{1}$ from the perspective of S:

S1: D does not recover the RTS or $\mathrm{S}$ does not recover the CTS, i.e., $\mathcal{E}_{\mathrm{R}} \cup\left(\mathcal{C}_{\mathrm{R}} \cap \mathcal{E}_{\mathrm{C}}\right)$ occurs. In either case, $\mathrm{S}$ has to wait for the CTS to arrive before attempting to recover it, so a cycle time of 2 time slots is always consumed. S1 occurs with probability $\operatorname{Pr}\left(\mathcal{E}_{\mathrm{R}}\right)+\operatorname{Pr}\left(\mathcal{C}_{\mathrm{R}}, \mathcal{E}_{\mathrm{C}}\right)=1-\operatorname{Pr}\left(\mathcal{C}_{\mathrm{R}}, \mathcal{C}_{\mathrm{C}}\right)$.

S2: $\quad S$ recovers the CTS, i.e., $\mathcal{C}_{\mathrm{R}} \cap \mathcal{C}_{\mathrm{C}}$. Hence, the DATA will be sent and a cycle time of $P+2$ slots will be consumed. S2 occurs with probability $\operatorname{Pr}\left(\mathcal{C}_{\mathrm{R}}, \mathcal{C}_{\mathrm{C}}\right)$.

On average, $P \operatorname{Pr}\left(\mathcal{C}_{\mathrm{D}}\right)$ slots are successfully transported from $S$ to $D$. Hence, the expected reward (in bits per cycle) is $\mathbb{E}[\mathcal{R}]=L_{\mathrm{sym}} R_{\mathrm{D}} P \operatorname{Pr}\left(\mathcal{C}_{\mathrm{D}}\right)$. Taking into account that situations $\mathrm{S} 1$ and $\mathrm{S} 2$ use 2 and $P+2$ slots, respectively, the expected cycle time (in slots) evaluates as $\mathbb{E}[\mathcal{T}]=2+$ $P \operatorname{Pr}\left(\mathcal{C}_{\mathrm{R}}, \mathcal{C}_{\mathrm{C}}\right)$. Using (7) and the above results for $\mathbb{E}[\mathcal{R}]$ and $\mathbb{E}[\mathcal{T}]$, the throughput (8) may be expressed as

$\bar{s}\left(R_{\mathrm{D}}, P\right)=\left[\frac{P \operatorname{Pr}\left(\mathcal{C}_{\mathrm{R}}, \mathcal{C}_{\mathrm{C}}\right)}{2+P \operatorname{Pr}\left(\mathcal{C}_{\mathrm{R}}, \mathcal{C}_{\mathrm{C}}\right)}\right] R_{\mathrm{D}} \operatorname{Pr}\left(\mathcal{C}_{\mathrm{D}} \mid \mathcal{C}_{\mathrm{R}}, \mathcal{C}_{\mathrm{C}} ; R_{\mathrm{D}}, P\right)$.

For clarity, $R_{\mathrm{D}}$ and $P$ are denoted explicitly as parameters in $\operatorname{Pr}\left(\mathcal{C}_{\mathrm{D}} \mid \mathcal{C}_{\mathrm{R}}, \mathcal{C}_{\mathrm{C}} ; R_{\mathrm{D}}, P\right)$. The fraction in (9) represents the fractional protocol overhead which increases monotonically and approaches one as $P \rightarrow \infty$. Hence, the asymptotic throughput is given by

$$
\overline{\bar{s}}\left(R_{\mathrm{D}}\right) \triangleq R_{\mathrm{D}} \lim _{P \rightarrow \infty} \operatorname{Pr}\left(\mathcal{C}_{\mathrm{D}} \mid \mathcal{C}_{\mathrm{R}}, \mathcal{C}_{\mathrm{C}} ; R_{\mathrm{D}}, P\right) .
$$

In particular for slot-by-slot detection, since the slots are decoded independently the DATA capture probability does not depend on $P$, so we have $\overline{\bar{s}}\left(R_{\mathrm{D}}\right)=R_{\mathrm{D}} \operatorname{Pr}\left(\mathcal{C}_{\mathrm{D}} \mid \mathcal{C}_{\mathrm{R}}, \mathcal{C}_{\mathrm{C}} ; R_{\mathrm{D}}\right)$.

\footnotetext{
${ }^{1}$ The definition of the end of a cycle from the perspective of $D$ can be different. For example, if D does not recover a RTS, the cycle is not even initiated as seen from D's perspective, while S has to wait for the CTS phase to be over (even without recovering any CTS) before it is sure that the cycle has terminated.
}

\section{Capture Probabilities: Detailed Analysis}

In Section IV-A, we model the traffic in the various phases of the RTS/CTS cycle as non-homogeneous Poisson processes. Then, Section IV-B derives the general expressions of the capture probabilities in different phases. Section IV-C relates these results to compute the desired conditional capture probabilities, eventually leading to the throughput (9). The analysis is conducted for the I.I.D channel, but is also relevant for the QS and QSNR channels, as discussed in Section IV-D.

\section{A. Traffic in Different Phases}

We are interested in the traffic conditioned on a successivecapture event $\mathcal{C}$ in the set $\mathcal{S}_{\mathcal{C}} \triangleq\left\{\emptyset, \mathcal{C}_{\mathrm{R}}, \mathcal{C}_{\mathrm{C}}, \mathcal{C}_{\mathrm{D}}\right\}$, or equivalently $\mathcal{S}_{\mathcal{C}}=\left\{\emptyset, \mathcal{C}_{\mathrm{R}}, \mathcal{C}_{\mathrm{R}} \cap \mathcal{C}_{\mathrm{C}}, \mathcal{C}_{\mathrm{R}} \cap \mathcal{C}_{\mathrm{C}} \cap \mathcal{C}_{\mathrm{D}}\right\}$. Here, the nonevent $\emptyset$ denotes that the protocol is just starting. For every phase $\Phi \in\{\mathrm{R}, \mathrm{C}, \mathrm{D}\}$, we denote

- $\mathcal{G}_{\Phi}(\boldsymbol{a} \mid \mathcal{C})$ as the conditional traffic intensity in phase $\Phi$ (i.e., when the $\Phi$ packet is sent), and

- $\mathcal{P}_{\Phi}(\boldsymbol{a} \mid \mathcal{C})$ as the conditional probability that an receiver at $\boldsymbol{a}$ recovers the $\Phi$ packet. This receiver can be an interferer, $\mathrm{S}$ or $\mathrm{D}$.

Both of the above functions give an quantitative picture of the network in the $\Phi$ phase: $\mathcal{G}_{\Phi}(\boldsymbol{a} \mid \mathcal{C})$ indicates the likelihood of having interference that originates from $\boldsymbol{a}$, while $\mathcal{P}_{\Phi}(\boldsymbol{a} \mid \mathcal{C})$ indicates the likelihood of receiving a packet at a. Our expressions imply a refined notion of hidden and exposed nodes, which we study from a probabilistic view. Specifically, a potentially harmful interferer remains uninhibited with probability $1-\mathcal{P}_{\mathrm{R}}\left(\boldsymbol{a} \mid \mathcal{C}_{\mathrm{R}}\right)$ in the CTS phase and with probability $\left(1-\mathcal{P}_{\mathrm{R}}\left(\boldsymbol{a} \mid \mathcal{C}_{\mathrm{R}}, \mathcal{C}_{\mathrm{C}}\right)\right)\left(1-\mathcal{P}_{\mathrm{C}}\left(\boldsymbol{a} \mid \mathcal{C}_{\mathrm{R}}, \mathcal{C}_{\mathrm{C}}\right)\right)$ in the DATA phase, while a harmless remote node is unjustly inhibited with probability $\mathcal{P}_{R}\left(\boldsymbol{a} \mid \mathcal{C}_{\mathrm{R}}\right)$ in the CTS phase and with probability $1-\left(1-\mathcal{P}_{\mathrm{R}}\left(\boldsymbol{a} \mid \mathcal{C}_{\mathrm{R}}, \mathcal{C}_{\mathrm{C}}\right)\right)\left(1-\mathcal{P}_{\mathrm{C}}\left(\boldsymbol{a} \mid \mathcal{C}_{\mathrm{R}}, \mathcal{C}_{\mathrm{C}}\right)\right)$ in the DATA phase.

Thus, the quantity $\mathcal{P}_{\Phi}(\boldsymbol{a} \mid \mathcal{C})$ is particularly interesting for cases when $\mathcal{C}$ denotes an event in the present and prior phases of the protocol (e.g., $\Phi=\mathrm{C}$ and $\mathcal{C}=\mathcal{C}_{\mathrm{R}} \cap \mathcal{C}_{\mathrm{C}}$ ) and $\boldsymbol{a}$ refers to a potential interferer. We do not include degenerate cases of $\mathcal{P}_{\Phi}(\boldsymbol{a} \mid \mathcal{C})=1$ when the capture event under consideration is the same or a subset of the conditioning event.

1) Distribution of $\mathbb{T}_{\mathrm{C}}$ : In the CTS phase, the interferers transmit if data have arrived and if they have not captured the RTS. Since $\mathcal{C}_{\mathrm{R}}$ has occurred, it is likely that the interference around D was small in the RTS phase. Potential CTS interferers that are near to D would also have captured the RTS with high probability and thus are not likely to transmit in the CTS phase. This leads to a lower traffic intensity $\mathcal{G}_{\mathrm{C}}\left(\boldsymbol{a} \mid \mathcal{C}_{\mathrm{R}}\right)$ for $\boldsymbol{a} \approx \boldsymbol{a}_{\mathrm{D}}$, i.e., for interferers that are close to D. Although the data arrival process stems from a Poisson point process, it is pruned by the RTS inhibition to give the data transmission process that describes the traffic.

To be specific, the probability that an interferer at $\boldsymbol{a}$ is captured, and hence is inhibited by the RTS, is given by $1-\mathcal{P}_{\mathrm{R}}\left(\boldsymbol{a} \mid \mathcal{C}_{\mathrm{R}}\right)$. By taking such inhibitions as independent, we can use the thinning property of the spatial Poisson process [23]. Simulation results lend support to this model. Since the original traffic intensity if there were no inhibition is $G_{o}$, 
the traffic in the CTS phase is a non-homogeneous Poisson process with traffic intensity

$$
\mathcal{G}_{\mathrm{C}}(\boldsymbol{a} \mid \mathcal{C})=G_{o}\left(1-\mathcal{P}_{\mathrm{R}}(\boldsymbol{a} \mid \mathcal{C})\right), \mathcal{C}=\mathcal{C}_{\mathrm{R}}
$$

This illustrates how an inhibition affects the traffic intensity in the network over time.

2) Distribution of $\mathbb{T}_{\mathrm{D}}$ : In the DATA phase, the interferer is not inhibited if it neither recovers the RTS nor the CTS. Conditioned on $\mathcal{C}=\mathcal{C}_{\mathrm{R}} \cap \mathcal{C}_{\mathrm{C}}$, this occurs with probability $\left(1-\mathcal{P}_{\mathrm{C}}(\boldsymbol{a} \mid \mathcal{C})\right)\left(1-\mathcal{P}_{\mathrm{R}}(\boldsymbol{a} \mid \mathcal{C})\right)$. Similarly, by taking the inhibitions as independent and using the thinning property, $\mathbb{T}_{\mathrm{D}}$ conditioned on $\mathcal{C}_{\mathrm{R}} \cap \mathcal{C}_{\mathrm{C}}$ is described by a non-homogeneous Poisson process with traffic intensity

$$
\mathcal{G}_{\mathrm{D}}(\boldsymbol{a} \mid \mathcal{C})=G_{o}\left(1-\mathcal{P}_{\mathrm{C}}(\boldsymbol{a} \mid \mathcal{C})\right)\left(1-\mathcal{P}_{\mathrm{R}}(\boldsymbol{a} \mid \mathcal{C})\right),
$$

where $\mathcal{C}=\mathcal{C}_{\mathrm{R}} \cap \mathcal{C}_{\mathrm{C}}$.

3) Generalization: Generally, we model the traffic conditioned on any $\mathcal{C} \in \mathcal{S}_{\mathcal{C}}$ by Poisson processes. In particular, the traffic in the CTS and DATA phase have traffic intensity (11) and (12), respectively, by replacing $\mathcal{C}$ accordingly, while in the RTS phase the traffic intensity $\mathcal{G}_{\mathrm{R}}(\boldsymbol{a} \mid \mathcal{C})$ will be determined subsequently.

\section{B. Capture Probability}

We consider a node at $\boldsymbol{a}_{\mathrm{tx}}$ that transmits a packet to a receiver at $\boldsymbol{a}_{\mathrm{rx}}$ at rate $R=2^{z}-1$. The traffic during transmission follows a Poisson process with traffic intensity $\mathcal{G}(\boldsymbol{a}), \boldsymbol{a} \in \mathcal{A}$. The capture event $\mathcal{C}_{\emptyset}$ is independent of past capture events (e.g., in the ALOHA protocol), but depends on $\mathcal{G}$. Its capture probability is given by [15]

$$
\begin{aligned}
& \operatorname{Pr}\left\{\mathcal{C}_{\emptyset} \mid \boldsymbol{a}_{\mathrm{tx}}, \boldsymbol{a}_{\mathrm{rx}}, z, \mathcal{G}\right\} \\
= & \int_{\mathbb{T}} \operatorname{Pr}\left\{\mathcal{C}_{\emptyset} \mid \boldsymbol{a}_{\mathrm{tx}}, \boldsymbol{a}_{\mathrm{rx}}, z, \mathbb{T}\right\} f_{\mathbb{T}}(\mathbb{T} \mid \mathcal{G}) \mathrm{d} \mathbb{T} \\
= & \exp \left(-\frac{z N_{\mathrm{o}}}{\bar{\gamma}}-\int_{\boldsymbol{a} \in \mathcal{A}} \mathcal{J}(\boldsymbol{a}) \mathrm{d} \boldsymbol{a}\right),
\end{aligned}
$$

where $\mathcal{J}(\boldsymbol{a}) \triangleq W\left(z,\left|\boldsymbol{a}_{\mathrm{tx}}-\boldsymbol{a}_{\mathrm{rx}}\right|,\left|\boldsymbol{a}-\boldsymbol{a}_{\mathrm{rx}}\right|\right) \mathcal{G}(\boldsymbol{a})$ and the vulnerability weight factor is

$$
W(z, x, y) \triangleq z x^{\beta} /\left(z x^{\beta}+y^{\beta}\right) .
$$

For the derivation of (13b) we refer to [15] for the case of ALOHA.

Next, we make use of (13) to find a generic expression for $\mathcal{P}_{\Phi}(\boldsymbol{a} \mid \mathcal{C}), \Phi=\mathrm{R}, \mathrm{C}, \mathrm{D}$. We will exploit the observation that given the traffic $\mathbb{T}_{\Phi}$, the capture probability of a receiver in phase $\Phi$ is independent of any event $\mathcal{C}$, i.e.,

$$
\mathcal{P}_{\Phi}\left(\boldsymbol{a} \mid \mathcal{C}, \mathbb{T}_{\Phi}\right)=\mathcal{P}_{\Phi}\left(\boldsymbol{a} \mid \mathbb{T}_{\Phi}\right) .
$$

This is because $\mathbb{T}_{\Phi}$ is sufficient to determine the capture probability, by using (4) for $\Phi=\mathrm{R}$ and $\Phi=\mathrm{C}$, or by using (5) or (6) for $\Phi=\mathrm{D}$.

As a shorthand, we denote $\mathcal{G}_{\Phi}(\boldsymbol{a} \mid \mathcal{C}), \boldsymbol{a} \in \mathcal{A}$, as $\mathcal{G}_{\Phi \mid \mathcal{C}}$. Given $\mathcal{C}$, the probability of capturing the $\Phi$ packet at $\boldsymbol{a}$ is

$$
\begin{aligned}
& \mathcal{P}_{\Phi}(\boldsymbol{a} \mid \mathcal{C})=\int_{\mathbb{T}_{\Phi}} \mathcal{P}_{\Phi}\left(\boldsymbol{a} \mid \mathcal{C}, \mathbb{T}_{\Phi}\right) f_{\mathbb{T}_{\Phi}}\left(\mathbb{T}_{\Phi} \mid \mathcal{G}_{\Phi \mid \mathcal{C}}\right) \mathrm{d} \mathbb{T}_{\Phi} \\
= & \int_{\mathbb{T}_{\Phi}} \mathcal{P}_{\Phi}\left(\boldsymbol{a} \mid \mathbb{T}_{\Phi}\right) f_{\mathbb{T}_{\Phi}}\left(\mathbb{T}_{\Phi} \mid \mathcal{G}_{\Phi \mid \mathcal{C}}\right) \mathrm{d} \mathbb{T}_{\Phi} \\
= & \int_{\mathbb{T}_{\Phi}} \operatorname{Pr}\left\{\mathcal{C}_{\emptyset} \mid \boldsymbol{a}_{\mathrm{tx}}, \boldsymbol{a}, z_{\Phi}, \mathbb{T}_{\Phi}\right\} f_{\mathbb{T}_{\Phi}}\left(\mathbb{T}_{\Phi} \mid \mathcal{G}_{\Phi \mid \mathcal{C}}\right) \mathrm{d} \mathbb{T}_{\Phi},
\end{aligned}
$$

where $a_{\mathrm{tx}}=a_{\mathrm{S}}$ for $\Phi=\mathrm{R}, \mathrm{D}$ and $\boldsymbol{a}_{\mathrm{tx}}=\boldsymbol{a}_{\mathrm{S}}$ for $\Phi=\mathrm{C}$. Here, (16a) follows from the law of total probability and (16b) follows from (15), (16c) follows from the definition of $\mathcal{P}_{\Phi}\left(\boldsymbol{a} \mid \mathbb{T}_{\Phi}\right)$. Comparing (16c) with (13a), we get

$$
\begin{aligned}
& \mathcal{P}_{\mathrm{R}}(\boldsymbol{a} \mid \mathcal{C})=\operatorname{Pr}\left\{\mathcal{C}_{\emptyset} \mid \boldsymbol{a}_{\mathrm{S}}, \boldsymbol{a}, z_{\mathrm{R}}, \mathcal{G}_{\mathrm{R} \mid \mathcal{C}}\right\}, \\
& \mathcal{P}_{\mathrm{C}}(\boldsymbol{a} \mid \mathcal{C})=\operatorname{Pr}\left\{\mathcal{C}_{\emptyset} \mid \boldsymbol{a}_{\mathrm{D}}, \boldsymbol{a}, z_{\mathrm{C}}, \mathcal{G}_{\mathrm{C} \mid \mathcal{C}}\right\}, \\
& \mathcal{P}_{\mathrm{D}}(\boldsymbol{a} \mid \mathcal{C})=\operatorname{Pr}\left\{\mathcal{C}_{\emptyset} \mid \boldsymbol{a}_{\mathrm{S}}, \boldsymbol{a}, z_{\mathrm{D}}, \mathcal{G}_{\mathrm{D} \mid \mathcal{C}}\right\} .
\end{aligned}
$$

These probabilities translate (13) to the results for the RTS/CTS protocol. Interestingly, the conditioning on the capture probability becomes a conditioning on the traffic intensity. We emphasize that the receiver considered in (17), with arbitrary location $\boldsymbol{a}$, can be an interferer, S or D.

\section{Relating Traffic Intensity and Capture Probability}

We now apply (17) to obtain $\operatorname{Pr}\left(\mathcal{C}_{\mathrm{R}}\right), \operatorname{Pr}\left(\mathcal{C}_{\mathrm{C}} \mid \mathcal{C}_{\mathrm{R}}\right)$ and $\operatorname{Pr}\left(\mathcal{C}_{\mathrm{D}} \mid \mathcal{C}_{\mathrm{R}}, \mathcal{C}_{\mathrm{C}}\right)$ in the DATA capture probability (7).

1) Capture Probability of RTS: In the RTS phase no inhibition is active yet, so (13) applies, in which case the receiver is $\mathrm{S}$, the transmitter ia $\mathrm{D}, z=z_{\mathrm{R}}$ and $\mathcal{G}(\boldsymbol{a})=G_{o}, \boldsymbol{a} \in \mathcal{A}$ :

$$
\operatorname{Pr}\left(\mathcal{C}_{\mathrm{R}}\right)=\exp \left(-z_{\mathrm{R}} N_{\circ} / \bar{\gamma}-a_{s}^{2} \pi^{2} G_{o} \sqrt{z_{\mathrm{R}}} / 2\right),
$$

with $a_{s}=\left|\boldsymbol{a}_{\mathrm{S}}-\boldsymbol{a}_{\mathrm{D}}\right|$. This closed-form expression corresponds to the results derived in [15].

2) Capture Probability of CTS: To find $\operatorname{Pr}\left(\mathcal{C}_{C} \mid \mathcal{C}_{R}\right)$, we apply (17b) by letting the receiver be $S$ (so $\boldsymbol{a}=\boldsymbol{a}_{\mathrm{S}}$ ) and $\mathcal{C}=\mathcal{C}_{\mathrm{R}}$ :

$$
\operatorname{Pr}\left(\mathcal{C}_{\mathrm{C}} \mid \mathcal{C}_{\mathrm{R}}\right)=\operatorname{Pr}\left\{\mathcal{C}_{\emptyset} \mid \boldsymbol{a}_{\mathrm{D}}, \boldsymbol{a}_{\mathrm{S}}, z_{\mathrm{C}}, \mathcal{G}_{\mathrm{C} \mid \mathcal{C}_{\mathrm{R}}}\right\} .
$$

Fig. 3 summarizes the subsequent calculations involved. To calculate $\mathcal{G}_{\mathrm{C}}\left(\boldsymbol{a} \mid \mathcal{C}_{\mathrm{R}}\right)$ in (19), we use (11), which in turn requires $\mathcal{P}_{\mathrm{R}}\left(\boldsymbol{a} \mid \mathcal{C}_{\mathrm{R}}\right), \boldsymbol{a} \in \mathcal{A}$. To compute $\mathcal{P}_{\mathrm{R}}\left(\boldsymbol{a} \mid \mathcal{C}_{\mathrm{R}}\right)$, we apply (17a) by letting the receiver be an interferer at $\boldsymbol{a}$, and letting $\mathcal{C}=$ $\mathcal{C}_{R}$. Finally, we require $\mathcal{G}_{\mathrm{R}}\left(\boldsymbol{a} \mid \mathcal{C}_{\mathrm{R}}\right)$ to be known. As derived in Appendix I, $\mathcal{G}_{\mathrm{R}}\left(\boldsymbol{a} \mid \mathcal{C}_{\mathrm{R}}\right)$ can be expressed as

$$
\mathcal{G}_{\mathrm{R}}\left(\boldsymbol{a} \mid \mathcal{C}_{\mathrm{R}}\right)=G_{o}\left(1-W\left(z_{\mathrm{R}}, a_{s},\left|\boldsymbol{a}-\boldsymbol{a}_{\mathrm{D}}\right|\right)\right) .
$$

This conditional traffic intensity can be interpreted as an $a$ posteriori traffic intensity, knowing $\mathcal{C}_{\mathrm{R}}$. It differs from the $a$ priori traffic intensity $\mathcal{G}_{\mathrm{R}}(\boldsymbol{a})=G_{o}$. Intuitively, we expect that given $\mathcal{C}_{\mathrm{R}}$ the amount of interference that occurred around $\mathrm{D}$ in the RTS phase was likely to be small - otherwise the RTS would not have captured D. From $W$ defined in (14), we see indeed that the traffic intensity (20) is small around D and approaches $G_{O}$ further away from D.

3) Capture Probability of DATA: We consider the DATA capture probability $\operatorname{Pr}\left(\mathcal{C}_{\mathrm{D}} \mid \mathcal{C}_{\mathrm{R}}, \mathcal{C}_{\mathrm{C}}\right)$ using either slot-by-slot or entire-packet DATA detection of Section II-D. We note that both approaches of detection are equivalent for unit DATA packet length, i.e., $P=1$.

For slot-by-slot packet detection, we apply (17c) by letting the receiver be $\mathrm{D}$ (so $\boldsymbol{a}=\boldsymbol{a}_{\mathrm{D}}$ ) and $\mathcal{C}=\mathcal{C}_{\mathrm{R}} \cap \mathcal{C}_{\mathrm{C}}$. We obtain

$$
\operatorname{Pr}\left(\mathcal{C}_{\mathrm{D}} \mid \mathcal{C}_{\mathrm{R}}, \mathcal{C}_{\mathrm{C}}\right)=\operatorname{Pr}\left\{\mathcal{C}_{\emptyset} \mid \boldsymbol{a}_{\mathrm{S}}, \boldsymbol{a}_{\mathrm{D}}, z_{\mathrm{D}}, \mathcal{G}_{\mathrm{D} \mid \mathcal{C}_{\mathrm{R}}, \mathcal{C}_{\mathrm{C}}}\right\}
$$

where the traffic intensity $\mathcal{G}_{\mathrm{D}}\left(\boldsymbol{a} \mid \mathcal{C}_{\mathrm{R}}, \mathcal{C}_{\mathrm{C}}\right)$ has been shown to be given by (12). Following the same arguments as earlier, we arrive at a sequence of computations summarized in Fig. 4. 


$$
\stackrel{(20)}{\longrightarrow} \mathcal{G}_{\mathrm{R}}\left(\boldsymbol{a} \mid \mathcal{C}_{\mathrm{R}}\right) \stackrel{(17 a)}{\mathcal{C}=\mathcal{C}_{\mathrm{R}}} \mathcal{P}_{\mathrm{R}}\left(\boldsymbol{a} \mid \mathcal{C}_{\mathrm{R}}\right) \stackrel{(11)}{\longrightarrow} \mathcal{G}_{\mathrm{C}}\left(\boldsymbol{a} \mid \mathcal{C}_{\mathrm{R}}\right) \stackrel{(19)}{\longrightarrow} \operatorname{Pr}\left(\mathcal{C}_{\mathrm{C}} \mid \mathcal{C}_{\mathrm{R}}\right)
$$

Fig. 3. Relationship of capture probabilities and traffic intensities over time and space in calculating $\operatorname{Pr}\left(\mathcal{C}_{\mathrm{C}} \mid \mathcal{C}_{\mathrm{R}}\right)$.

$$
\begin{aligned}
& \stackrel{(22 a)}{\longrightarrow} \mathcal{G}_{\mathrm{C}}\left(\boldsymbol{a} \mid \mathcal{C}_{\mathrm{R}}, \mathcal{C}_{\mathrm{C}}\right) \underset{\mathcal{C}=\mathcal{C}_{\mathrm{R}} \cap \mathcal{C}_{\mathrm{C}}}{\longrightarrow} \mathcal{P}_{\mathrm{C}}\left(\boldsymbol{a} \mid \mathcal{C}_{\mathrm{R}}, \mathcal{C}_{\mathrm{C}}\right) \stackrel{(12)}{\longrightarrow} \mathcal{G}_{\mathrm{D}}\left(\boldsymbol{a} \mid \mathcal{C}_{\mathrm{R}}, \mathcal{C}_{\mathrm{C}}\right) \stackrel{(21)}{\longrightarrow} \operatorname{Pr}\left(\mathcal{C}_{\mathrm{D}} \mid \mathcal{C}_{\mathrm{R}}, \mathcal{C}_{\mathrm{C}}\right) \\
& \stackrel{(22 b)}{\longrightarrow} \mathcal{G}_{\mathrm{R}}\left(\boldsymbol{a} \mid \mathcal{C}_{\mathrm{R}}, \mathcal{C}_{\mathrm{C}}\right) \stackrel{(17 a)}{\underset{\mathcal{C}=\mathcal{C}_{\mathrm{R}} \cap \mathcal{C}_{\mathrm{C}}}{\longrightarrow}} \mathcal{P}_{\mathrm{R}}\left(\boldsymbol{a} \mid \mathcal{C}_{\mathrm{R}}, \mathcal{C}_{\mathrm{C}}\right)^{(12)}
\end{aligned}
$$

Fig. 4. Relationship of capture probabilities and traffic intensities over time and space in calculating $\operatorname{Pr}\left(\mathcal{C}_{\mathrm{D}} \mid \mathcal{C}_{\mathrm{R}}, \mathcal{C}_{\mathrm{C}}\right)$.

Finally, what is left to compute are the a posteriori traffic intensities

$$
\begin{aligned}
& \mathcal{G}_{\mathrm{C}}\left(\boldsymbol{a} \mid \mathcal{C}_{\mathrm{R}}, \mathcal{C}_{\mathrm{C}}\right)=\left(1-W\left(z_{\mathrm{C}}, a_{s},\left|\boldsymbol{a}-\boldsymbol{a}_{\mathrm{S}}\right|\right)\right) \mathcal{G}_{\mathrm{C}}\left(\boldsymbol{a} \mid \mathcal{C}_{\mathrm{R}}\right), \\
& \mathcal{G}_{\mathrm{R}}\left(\boldsymbol{a} \mid \mathcal{C}_{\mathrm{R}}, \mathcal{C}_{\mathrm{C}}\right)=\left(1-W\left(z_{\mathrm{C}}, a_{s},\left|\boldsymbol{a}-\boldsymbol{a}_{\mathrm{S}}\right|\right)\right) \mathcal{G}_{\mathrm{R}}\left(\boldsymbol{a} \mid \mathcal{C}_{\mathrm{R}}\right),
\end{aligned}
$$

where the derivations are found in the Appendix II. Here, $\mathcal{G}_{\mathrm{C}}\left(\boldsymbol{a} \mid \mathcal{C}_{\mathrm{R}}\right)$ and $\mathcal{G}_{\mathrm{R}}\left(\boldsymbol{a} \mid \mathcal{C}_{\mathrm{R}}\right)$ are available from (11) and (20), respectively.

For entire-packet detection of DATA, the capture of the packet, or equivalently of a slot in the packet, is governed by (6). For I.I.D. channels, as the packet length $P$ increases, the distribution of the averaged mutual information $\overline{\mathcal{I}}_{\mathrm{D}}$ converges to a Gaussian distribution with mean $\mu=\mathbb{E}\left[\mathcal{I}_{\mathrm{D}, k} \mid \mathcal{C}_{\mathrm{R}}, \mathcal{C}_{\mathrm{C}}\right]$ and variance $\sigma^{2}=\left(\mathbb{E}\left[\mathcal{I}_{\mathrm{D}, k}^{2} \mid \mathcal{C}_{\mathrm{R}}, \mathcal{C}_{\mathrm{C}}\right]-\mu^{2}\right) / P$. For our RTS/CTS protocol, we have validated in simulations that this Gaussian approximation is fairly accurate even for small $P$, say $P=2$ (also see Fig. 8). The conditional pdf of $\mathcal{I}_{\mathrm{D}}$ (we drop the time index $k$ ) can be calculated from (21a) which can be written alternatively as $\operatorname{Pr}\left(\mathcal{I}_{\mathrm{D}}>2^{z_{\mathrm{D}}}-1 \mid \mathcal{C}_{\mathrm{R}}, \mathcal{C}_{\mathrm{C}}\right)$. Hence, $\mu$ and $\sigma_{\mathcal{I}}^{2}$ can also be computed. The capture probability is then approximated as

$$
\operatorname{Pr}\left(\mathcal{C}_{\mathrm{D}} \mid \mathcal{C}_{\mathrm{R}}, \mathcal{C}_{\mathrm{C}}\right) \approx Q\left(\frac{R_{\mathrm{D}}-\mu}{\sigma_{\mathcal{I}}}\right)
$$

where $Q(x)=\int_{x}^{\infty} \exp \left(-y^{2} / 2\right) / \sqrt{2 \pi} \mathrm{d} y$ is the Gaussian error integral function. In particular, consider the case that $P \rightarrow \infty$. By the law of large number $\overline{\mathcal{I}}_{\mathrm{D}}$ approaches $\mu$, a constant. Hence, a DATA packet is recovered if and only if its rate $R_{\mathrm{D}}$ is less than $\overline{\mathcal{I}}_{\mathrm{D}}$. It follows that the throughput (10) increases linearly with $R_{\mathrm{D}}$ for $R_{\mathrm{D}}<\mu$, but abruptly drops to 0 for $R_{\mathrm{D}} \geq \mu$.

Some of the conditional probabilities and traffic intensities depicted in Fig. 3 and Fig. 4 do not yield closed-form solutions. However, numerical results have been obtained. Intermediate results are presented in this paper in the form of contour plots of $\mathcal{G}_{\mathrm{C}}\left(\boldsymbol{a} \mid \mathcal{C}_{\mathrm{R}}\right), \mathcal{G}_{\mathrm{D}}\left(\boldsymbol{a} \mid \mathcal{C}_{\mathrm{R}}, \mathcal{C}_{\mathrm{C}}\right)$ in Fig. 5 indicating the extent of the RTS and CTS reservations.

\section{DATA Capture Probabilities in Different Channels}

We consider slot-by-slot DATA packet detection. The conditional DATA capture probability given $\mathbb{T}_{\text {cycle }}$ for the QS, QSNR and I.I.D. channels are related as follows:

$$
\begin{aligned}
\operatorname{Pr}\left(\mathcal{C}_{\mathrm{D}} \mid \mathbb{T}_{\text {cycle }} ; \text { I.I.D. }\right) & \leq \operatorname{Pr}\left(\mathcal{C}_{\mathrm{D}} \mid \mathbb{T}_{\text {cycle }} ; \mathrm{QSNR}\right) \\
& \leq \operatorname{Pr}\left(\mathcal{C}_{\mathrm{D}} \mid \mathbb{T}_{\text {cycle }} ; \mathrm{QS}\right) .
\end{aligned}
$$

Further, this relationship holds without conditioning on the traffic:

$$
\operatorname{Pr}\left(\mathcal{C}_{\text {D }} ; \text { I.I.D. }\right) \leq \operatorname{Pr}\left(\mathcal{C}_{\mathrm{D}} ; \text { QSNR }\right) \leq \operatorname{Pr}\left(\mathcal{C}_{\mathrm{D}} ; \text { QS }\right) .
$$

The proofs for (24) and (25) are given in Appendix III and Appendix IV, respectively. Although we cannot find analytical solutions of the cycle capture probabilities for the QS and QSNR channels, an analysis for the I.I.D. channel provides a lower bound on the capture probabilities. For comparison, results for the other channels are obtained by simulations.

For entire-packet DATA detection, on the other hand, an inequality such as (25) cannot be established; simulation results confirm that the relationship of the capture probabilities depend generally on $P$ (see Figure 8).

\section{Numerical Results}

For the I.I.D. channels, we present numerical evaluations of the analytical expressions in Section III. Monte Carlo simulations are conducted for other types of channels. We considered the noise-free case $N_{\mathrm{o}}=0$ to isolate the effect of interference and we used the following system parameters: $\boldsymbol{a}_{\mathrm{D}}=[0,0], \boldsymbol{a}_{\mathrm{S}}=[0.5,0], G_{o}=1 / \pi$ ppsa. In our simulations, we used a square of length 20 centered at the origin for the operation region $\mathcal{A}$; this region is sufficiently large to simulate an infinite operation region. The RTS and CTS rates are set to $R_{\mathrm{R}}=R_{\mathrm{C}}=1 \mathrm{bit} / \mathrm{symbol}$, corresponding for instance to using QPSK modulation with a code rate of $1 / 2$.

\section{A. Traffic Intensity}

As no spatial reservation is in place before the RTS, the starting traffic intensity is uniform with $G_{o}=1 / \pi$. Fig. 5 shows the normalized traffic intensity as the protocol progresses through the CTS and DATA phases. The traffic during the CTS period $\mathcal{G}_{\mathrm{C}}\left(\boldsymbol{a} \mid \mathcal{C}_{\mathrm{R}}\right)$ reduces mostly in the vicinity of $\mathrm{S}$. This reduction arises because the RTS performs a spatial reservation around $\mathrm{S}$, though with fuzzy boundaries. As a result, the CTS capture probability improves. After the RTS is recovered, D transmits a CTS which reserves the channel during the DATA period. This second reservation is centered around D, but jointly with the first reservation of the RTS, creates an enlarged, overall region of reservation as indicated by $\mathcal{G}_{\mathrm{D}}\left(\boldsymbol{a} \mid \mathcal{C}_{\mathrm{R}}, \mathcal{C}_{\mathrm{C}}\right)$. Hence, the DATA capture probability also improves.

The use of RTS for inhibition in the DATA phase may be inappropriate, since the inhibition is centered around $S$ rather than D which acts as the receiver in the DATA phase. Alternatively, the RTS inhibition can be eliminated by reducing 


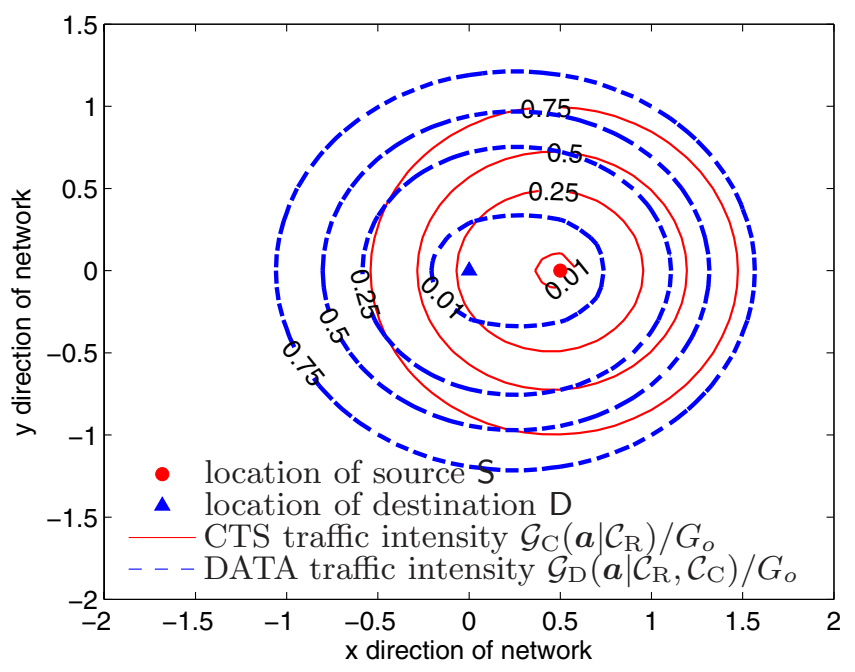

Fig. 5. Contour plots of normalized traffic intensities in the CTS and DATA phases. The contours illustrate the spatial effects of inhibition by the RTS and CTS packets. In the CTS phase, only the RTS creates a spatial reservation around S. In the DATA phase, both the RTS and CTS are used, which creates a larger reservation area around $\mathrm{S}$ and $\mathrm{D}$.

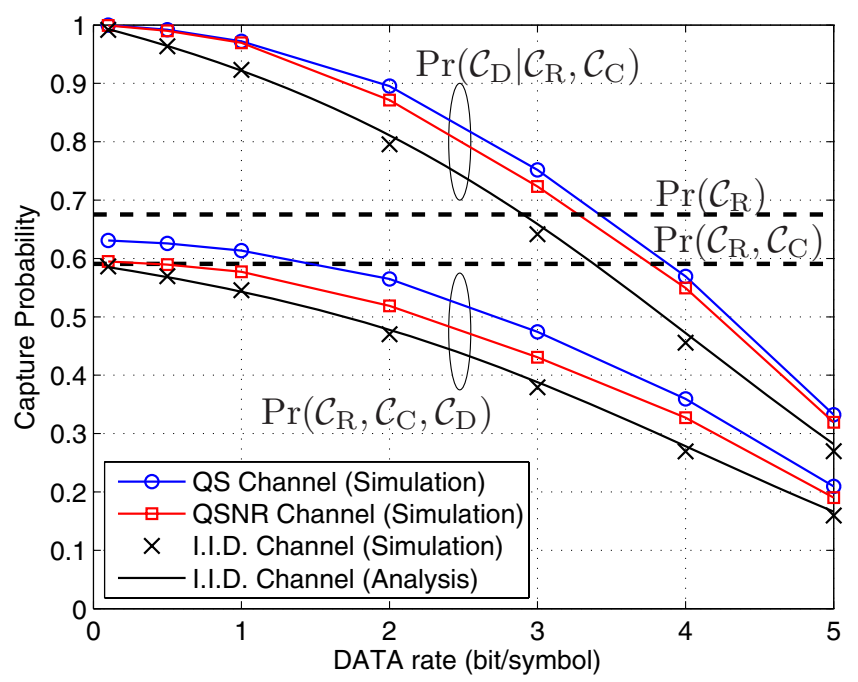

Fig. 6. The conditional DATA capture probability $\operatorname{Pr}\left(\mathcal{C}_{\mathrm{D}} \mid \mathcal{C}_{\mathrm{R}}, \mathcal{C}_{\mathrm{C}}\right)$ and DATA capture probability $\operatorname{Pr}\left(\mathcal{C}_{\mathrm{R}}, \mathcal{C}_{\mathrm{C}}, \mathcal{C}_{\mathrm{D}}\right)$ for different channels. The RTS capture probability $\operatorname{Pr}\left(\mathcal{C}_{\mathrm{R}}\right)$ and the probability that the RTS and CTS are both captured, $\operatorname{Pr}\left(\mathcal{C}_{\mathrm{R}}, \mathcal{C}_{\mathrm{C}}\right)$, are also plotted. Here, $P=1$ is used.

its inhibition window; this has been considered separately in [24]. Whether it is preferable to have a reduced or increased inhibition window is a tradeoff, in particular, between the DATA capture probability for the users of the RTS/CTS protocol, and the penalty for inhibiting users of the ALOHA protocol.

\section{B. Capture Probability}

The cycle capture probability $\operatorname{Pr}\left(\mathcal{C}_{\mathrm{D}}\right)=\operatorname{Pr}\left(\mathcal{C}_{\mathrm{R}}, \mathcal{C}_{\mathrm{C}}, \mathcal{C}_{\mathrm{D}}\right)$ and the conditional DATA capture probability $\operatorname{Pr}\left(\mathcal{C}_{\mathrm{D}} \mid \mathcal{C}_{\mathrm{R}}, \mathcal{C}_{\mathrm{C}}\right)$ have been plotted in Fig. 6 against the DATA rate $R_{\mathrm{D}}$. We use $P=1$ and so the capture probabilities are the same for both slot-by-slot and entire-packet DATA detection. Monte Carlo simulations have been carried out for the I.I.D., QSNR and QS channels using 10,000 RTS/CTS cycles. We observe that the simulation results match the analytical results for the I.I.D. channels, validating the accuracy of modeling the CTS and PAY traffic as Poisson processes. Next, we observe that the capture probabilities for the QS and QSNR channels are larger than the I.I.D. channel, as predicted in the analysis. For example, at a rate of $R_{\mathrm{D}}=2$, the QS channel is about $20 \%$ higher in $\operatorname{Pr}\left(\mathcal{C}_{\mathrm{D}}\right)$ than the I.I.D. channel.

Fig. 6 also serves to compare our results with those resulting from the commonly made assumption that $\operatorname{Pr}\left(\mathcal{C}_{\mathrm{R}}, \mathcal{C}_{\mathrm{C}}, \mathcal{C}_{\mathrm{D}}\right) \approx$ $\operatorname{Pr}\left(\mathcal{C}_{R}\right)$. This approximation is realistic only if the DATA rate is equally low or lower than the rate of the RTS or CTS rates (i.e., 1 bit/symbol), which in practice is not the case. For the I.I.D. channel, for instance, we see that $\operatorname{Pr}\left(\mathcal{C}_{R}, \mathcal{C}_{C}, \mathcal{C}_{\mathrm{D}}\right) \ll$ $\operatorname{Pr}\left(\mathcal{C}_{R}\right)=0.68$ for sufficiently large DATA rates. So we conclude that $\operatorname{Pr}\left(\mathcal{C}_{R}\right)$ (indicated by a dotted line) is overly optimistic. Even $\operatorname{Pr}\left(\mathcal{C}_{\mathrm{R}}, \mathcal{C}_{\mathrm{C}}\right)=0.59$ is optimistic for the cycle capture probability, particularly at high DATA rates, say larger than $2 \mathrm{bit} / \mathrm{symbol}$. These observations justify our effort to explicitly model the probabilistic effects of successive captures and to determine their probabilities.

\section{Throughput}

To illustrate the potential of rate optimization, we take $R_{\mathrm{D}}$ to be continuous. In practice, rates are discrete. We optimize $R_{\mathrm{D}}$ to maximize the link throughput for a fixed RTS and CTS rates, and compare this with slotted ALOHA transmissions, thus without RTS/CTS. For ALOHA transmission at rate $R_{\mathrm{ALOHA}}$, the traffic intensity is always $G_{0}$. Hence the ALOHA capture probability is found from (18) by replacing $z_{\mathrm{R}}$ with $2^{R_{\mathrm{ALOHA}}}-1$, and the throughput follows from multiplying $R_{\mathrm{ALOHA}}$ with this ALOHA capture probability. For simplicity, we focus on only the I.I.D. and QS channels.

1) Slot-by-Slot DATA Detection: We first consider the throughput using slot-by-slot DATA detection. Fig. 7 shows the throughput obtained by substituting the simulated or analytical capture probabilities into the analytical expression (9). We observe that the throughput in the QS channel is larger than in the I.I.D. channel, which is expected because the DATA capture probability in the QS channel has been shown to be larger. For fixed $P$ and increasing $R_{\mathrm{D}}, \bar{s}$ increases initially, because transmitting at a (too) low $R_{\mathrm{D}}$ limits the throughput. At sufficiently high $R_{\mathrm{D}}$, the throughput starts to reduce, due to reduced robustness against interference. We observe that the same optimum DATA rate maximizes the throughout for any $P$. This is because the DATA capture probability is independent of $P$ for slot-by-slot DATA detection.

Fig. 7 shows that for sufficiently large $P$ the RTS/CTS protocol outperforms ALOHA, at any common DATA rate. For moderate $P$, say $P=10$, if $R_{\mathrm{D}}<0.75$, the ALOHA throughput can marginally outperform that of the RTS/CTS; but if $R_{\mathrm{D}} \approx 3$, substantial throughput gain can be realized. Finally, if $P$ is too small, say $P=2$, then the overhead of the RTS/CTS protocol is too high, and even with properly tuned DATA rate, the throughput is comparable or less than using the ALOHA protocol.

2) Entire-Packet DATA Detection: Next we consider the throughput using entire-packet DATA detection. From Fig. 8, we observe that the simulation results for the I.I.D. channel 


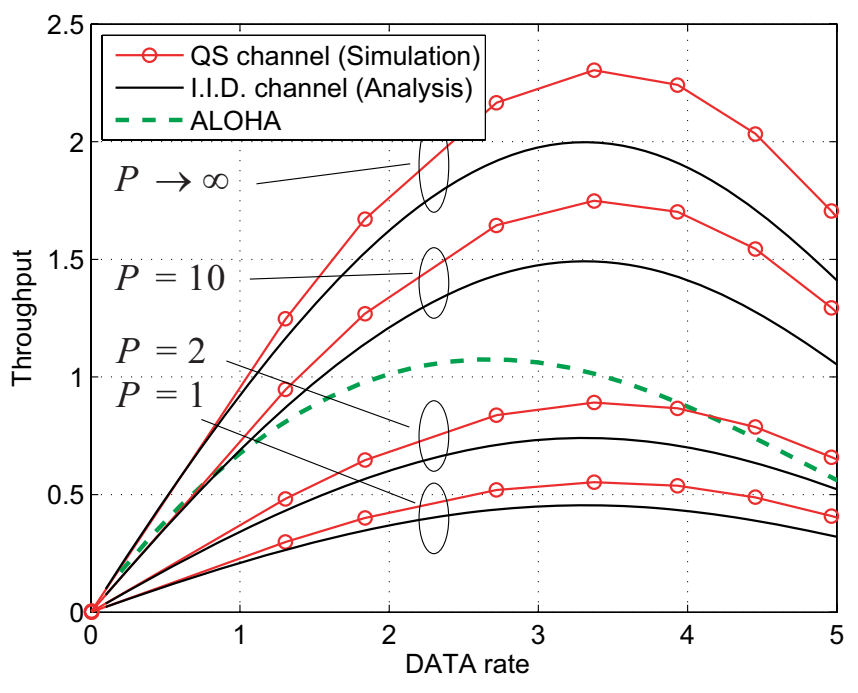

Fig. 7. Slot-by-slot DATA detection: throughput $\bar{s}$ vs rate of DATA slots $R_{\mathrm{D}}$ for various DATA slot length $P$.

match the analysis fairly well, despite the approximations used in (23). For $P=1,2$, we observe that the throughput for the QS channel is larger than for the I.I.D. channel, but as $P$ increases, the throughput for the I.I.D. channel becomes larger than for the QS channel ${ }^{2}$. In particular, Fig. 8 shows a distinct sharp optimum when $P \rightarrow \infty$, as the law of large number on the averaged mutual information takes effect. Hence, the entire packet is recovered if and only if $R_{\mathrm{D}} \leq \mu \approx 3.8 \mathrm{bit} / \mathrm{symbol}$. Similar to slot-by-slot DATA detection, for moderate or higher $P$, using the RTS/CTS protocol realizes a higher throughput by transmitting the DATA at around three times the RTS and CTS rates.

Generally, entire-packet DATA detection outperforms slotby-slot DATA detection, especially for large $P$. When $P \rightarrow \infty$ and for I.I.D channel, the maximum throughput is close to two times higher. However, for the QS channel, the maximum throughput of using entire-packet DATA detection is only marginally better. We conclude that in an I.D.D. channel entire-packet DATA detection can be used to significantly improve the throughput, but in a QS channel this detection may not be worthwhile, particularly as a substantially higher delay is incurred when decoding the packet as a whole.

Finally, Fig. 9 considers how the maximum throughput of the RTS/CTS and ALOHA protocols behave with distance $a_{s}$, using independently optimized DATA rates. We consider $P \rightarrow \infty$ and entire-packet DATA detection in an I.I.D. channel for the RTS/CTS protocol. As expected, both maximum throughputs decrease with $a_{s}$, but the rate of decrement is slower using the RTS/CTS protocol. At large $a_{s}$, using the RTS/CTS protocol can deliver up to five times the ALOHA throughput. Hence, the use of the RTS/CTS protocol is especially important when the source and destination are far apart.

\section{CONCLUSION}

We developed a mathematical framework for the behavior of the RTS/CTS protocol based on successive captures. In partic-

\footnotetext{
${ }^{2}$ The throughput for the QS channel for $P \rightarrow \infty$ is obtained by a semianalytical method. For completeness, the details are given in Appendix V.
}

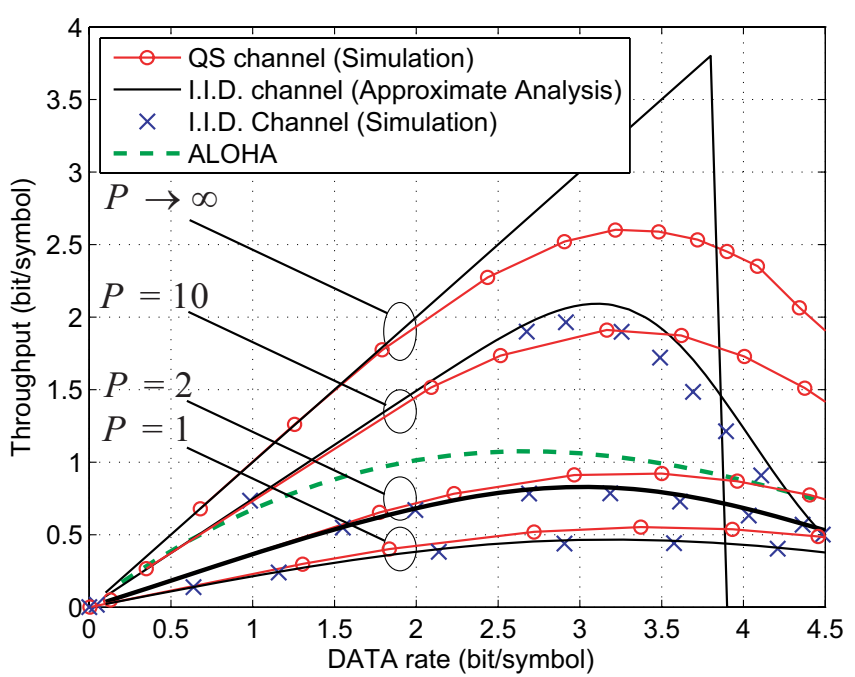

Fig. 8. Entire-packet DATA detection: throughput $\bar{s}$ vs rate of DATA slots $R_{\mathrm{D}}$ for various DATA slot length $P$.

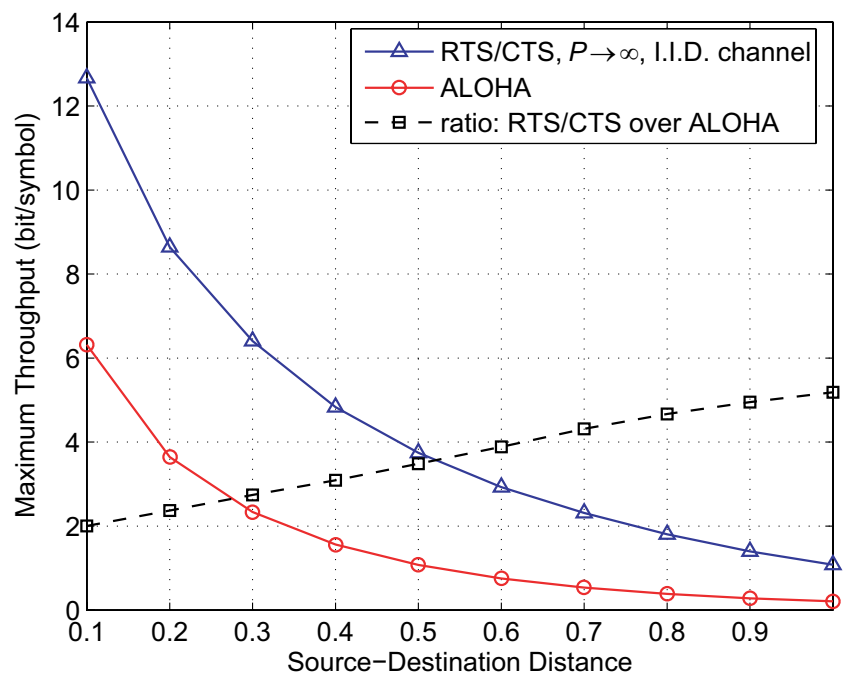

Fig. 9. Maximum throughput achieved with varying source-destination distance by using RTS/CTS protocol and ALOHA protocol.

ular we studied the evolution of the interfering traffic densities for successive phases in the protocol cycle. This allowed us to derive new expressions for the capture probabilities of the RTS, CTS and data packets. The statistical dependence of the capture probabilities has been calculated considering a chain of capture probabilities on the desired link, probabilities of potential interferers missing the inhibition messages, and the resulting interfering traffic intensity. We have shown that for ad-hoc networks, the popular assumption that the capture probability of the RTS is representative for the probability of a successful cycle is no longer accurate when we perform rate optimization.

Numerical results suggest that the RTS/CTS rates are preferably sent at a rate that is around three times lower than for the data payload; this significantly improves throughput. We observe that using the RTS/CTS protocol is especially worthwhile, as compared to the ALOHA protocol, when the data length is long and the source is far from the destination. 


\section{APPENDIX I}

\section{DERIVATION OF (20)}

We consider the RTS phase. We divide the operating region $\mathcal{A}$ into $K(\rightarrow \infty)$ non-overlapping regions, $\mathcal{A}_{k}$, each of area $\delta A(\rightarrow 0)$. An interferer at $\boldsymbol{a}_{k} \in \mathcal{A}_{k}$ transmits with probability $\operatorname{Pr}\left(k_{\mathrm{R}}\right.$ on $), k=1, \cdots, K$. By definition of the Poisson process, the traffic intensity in the RTS phase can be expressed as

$$
\mathcal{G}_{\mathrm{R}}\left(\boldsymbol{a}_{k}\right)=\lim _{\delta A \rightarrow 0} \frac{\operatorname{Pr}(k \text { on })}{\delta A} .
$$

Knowing that the RTS is recovered by the D, the a posteriori traffic intensity is then

$$
\begin{aligned}
\mathcal{G}_{\mathrm{R}}\left(\boldsymbol{a}_{k} \mid \mathcal{C}_{\mathrm{R}}\right) & =\lim _{\delta A \rightarrow 0} \frac{\operatorname{Pr}\left(k \text { on } \mid \mathcal{C}_{\mathrm{R}}\right)}{\delta A} \\
& =\lim _{\delta A \rightarrow 0} \frac{\operatorname{Pr}\left(k \text { on } \mid \mathcal{C}_{\mathrm{R}}\right)}{\operatorname{Pr}(k \text { on })} \mathcal{G}_{\mathrm{R}}\left(\boldsymbol{a}_{k}\right),
\end{aligned}
$$

by substituting (26). It is shown in [15, (29)] that

$$
\frac{\operatorname{Pr}\left(k \text { on } \mid \mathcal{C}_{\mathrm{R}}\right)}{\operatorname{Pr}(k \text { on })}=\frac{1-W\left(z_{\mathrm{R}}, a_{s},\left|\boldsymbol{a}_{k}-\boldsymbol{a}_{\mathrm{D}}\right|\right)}{1-W\left(z_{\mathrm{R}}, a_{s},\left|\boldsymbol{a}_{k}-\boldsymbol{a}_{\mathrm{D}}\right|\right) \operatorname{Pr}(k \text { on })},
$$

where $a_{s}=\left|\boldsymbol{a}_{\mathrm{S}}-\boldsymbol{a}_{\mathrm{D}}\right|$. Note that $\lim _{\delta A \rightarrow 0} \operatorname{Pr}(k$ on $)=0$; otherwise $\mathcal{G}_{\mathrm{R}}\left(\boldsymbol{a}_{k} \mid \mathcal{C}_{\mathrm{R}}\right) \rightarrow \infty$ in (26). So, (28) converges to $1-W\left(z_{\mathrm{R}}, a_{s},\left|\boldsymbol{a}_{k}-\boldsymbol{a}_{\mathrm{D}}\right|\right)$ as $\delta A \rightarrow 0$. Hence, (27) becomes $\mathcal{G}_{\mathrm{R}}\left(\boldsymbol{a}_{k} \mid \mathcal{C}_{\mathrm{R}}\right)=\left(1-W\left(z_{\mathrm{R}}, a_{s},\left|\boldsymbol{a}_{k}-\boldsymbol{a}_{\mathrm{D}}\right|\right)\right) G_{o}$ since $\mathcal{G}_{\mathrm{R}}(\boldsymbol{a})=$ $G_{o}$, hence completing the derivation.

\section{APPENDIX II}

\section{DERIVATION OF (22)}

We use similar arguments as in Appendix I to derive (22a), (22b). To show (22a), we consider the CTS phase and focus on an interferer at $\boldsymbol{a}_{k}$ that occupies a small area $\delta A$. The event " $k$ on" represents that interferer $k$ transmits in the CTS phase. By definition, the traffic intensity given $\mathcal{C}_{\mathrm{R}}$ is

$$
\mathcal{G}_{\mathrm{C}}\left(\boldsymbol{a}_{k} \mid \mathcal{C}_{\mathrm{R}}\right)=\lim _{\delta A \rightarrow 0} \frac{\operatorname{Pr}\left(k \text { on } \mid \mathcal{C}_{\mathrm{R}}\right)}{\delta A} .
$$

In the CTS phase, the traffic intensity at $\boldsymbol{a}_{k}$ given $\mathcal{C}_{\mathrm{R}}, \mathcal{C}_{\mathrm{C}}$ is

$$
\begin{aligned}
\mathcal{G}_{\mathrm{C}}\left(\boldsymbol{a}_{k} \mid \mathcal{C}_{\mathrm{R}}, \mathcal{C}_{\mathrm{C}}\right) & =\lim _{\delta A \rightarrow 0} \frac{\operatorname{Pr}\left(k \text { on } \mid \mathcal{C}_{\mathrm{R}}, \mathcal{C}_{\mathrm{C}}\right)}{\delta A} \\
& =\lim _{\delta A \rightarrow 0} \frac{\operatorname{Pr}\left(k \text { on } \mid \mathcal{C}_{\mathrm{C}}, \mathcal{C}_{\mathrm{R}}\right)}{\operatorname{Pr}\left(k \text { on } \mid \mathcal{C}_{\mathrm{R}}\right)} \mathcal{G}_{\mathrm{C}}\left(\boldsymbol{a}_{k} \mid \mathcal{C}_{\mathrm{R}}\right)
\end{aligned}
$$

by using (29). Similar to the derivations in [15, (29)], we get

$$
\frac{\operatorname{Pr}\left(k \text { on } \mid \mathcal{C}_{\mathrm{C}}, \mathcal{C}_{\mathrm{R}}\right)}{\operatorname{Pr}\left(k \text { on } \mid \mathcal{C}_{\mathrm{R}}\right)}=\frac{1-W\left(z_{\mathrm{C}}, a_{S},\left|\boldsymbol{a}_{k}-\boldsymbol{a}_{\mathrm{S}}\right|\right)}{1-W\left(z_{\mathrm{C}}, a_{S},\left|\boldsymbol{a}_{k}-\boldsymbol{a}_{\mathrm{S}}\right|\right) \operatorname{Pr}\left(k \text { on } \mid \mathcal{C}_{\mathrm{R}}\right)}
$$

which approaches its numerator as $\delta A \rightarrow 0$, and hence we obtain (22a).

To obtain (22b), we consider instead the RTS phase, i.e., the event " $k$ on" represents that interferer $k$ transmits in the RTS phase. By definition, the traffic intensity given $\mathcal{C}_{\mathrm{R}}, \mathcal{C}_{\mathrm{C}}$ is

$$
\begin{aligned}
\mathcal{G}_{\mathrm{R}}\left(\boldsymbol{a}_{k} \mid \mathcal{C}_{\mathrm{R}}, \mathcal{C}_{\mathrm{C}}\right) & =\lim _{\delta A \rightarrow 0} \frac{\operatorname{Pr}\left(k \text { on } \mid \mathcal{C}_{\mathrm{R}}, \mathcal{C}_{\mathrm{C}}\right)}{\delta A} \\
& =\lim _{\delta A \rightarrow 0} \frac{\operatorname{Pr}\left(k \text { on } \mid \mathcal{C}_{\mathrm{R}}\right)}{\delta A} \frac{\operatorname{Pr}\left(k \text { on } \mid \mathcal{C}_{\mathrm{C}}, \mathcal{C}_{\mathrm{R}}\right)}{\operatorname{Pr}\left(k \text { on } \mid \mathcal{C}_{\mathrm{R}}\right)} \\
& =\mathcal{G}_{\mathrm{R}}\left(\boldsymbol{a}_{k} \mid \mathcal{C}_{\mathrm{R}}\right)\left(1-W\left(z_{\mathrm{C}}, a_{S},\left|\boldsymbol{a}_{k}-\boldsymbol{a}_{\mathrm{S}}\right|\right)\right)
\end{aligned}
$$

by substituting (27) and following the same derivation as for (31). Thus, we obtain (22b).

\section{APPENDIX III}

\section{DERIVATION OF (24)}

The conditional DATA capture probability is given by

$\operatorname{Pr}\left(\mathcal{C}_{\mathrm{D}} \mid \mathbb{T}_{\text {cycle }}\right)=\operatorname{Pr}\left(\frac{\gamma_{\mathrm{R}}^{\text {sig }}}{\gamma_{\mathrm{R}}^{\text {int }}}>z_{\mathrm{R}}, \frac{\gamma_{\mathrm{C}}^{\text {sig }}}{\gamma_{\mathrm{C}}^{\text {int }}}>z_{\mathrm{C}}, \frac{\gamma_{\mathrm{D}}^{\text {sig }}}{\gamma_{\mathrm{D}}^{\text {int }}}>z_{\mathrm{D}} \mid \mathbb{T}_{\text {cycle }}\right)$

for any channel, by using (4) and (5). For conciseness, we denote the signal powers as $\gamma^{\text {sig }} \triangleq\left[\gamma_{R}^{\text {sig }}, \gamma_{C}^{\text {sig }}, \gamma_{D}^{\text {sig }}\right]$ and the total interference powers as $\gamma^{\text {int }} \triangleq\left[\gamma_{R}^{\text {int }}, \gamma_{C}^{\text {int }}, \gamma_{D}^{\text {int }}\right]$. Further, we denote the dummy variables used for integration as $\boldsymbol{y}=$ $\left[y_{1}, y_{2}, y_{3}\right], \boldsymbol{x}=\left[x_{1}, x_{2}, x_{3}\right]$. By an appropriate change of variables, (32) can be written as

$$
\operatorname{Pr}\left(\mathcal{C}_{\mathrm{D}} \mid \mathbb{T}_{\text {cycle }}\right)=\int_{0}^{\infty} \int_{0}^{\infty} \int_{0}^{\infty} f_{\boldsymbol{\gamma}^{\text {int }}}\left(\boldsymbol{x} \mid \mathbb{T}_{\text {cycle }}\right) \mathcal{K}(\boldsymbol{x}) \mathrm{d} \boldsymbol{x}
$$

where we define

$$
\mathcal{K}(\boldsymbol{x}) \triangleq \int_{y_{1}=z_{\mathrm{R}} x_{1}}^{\infty} \int_{y_{2}=z_{\mathrm{C}} x_{3}}^{\infty} \int_{y_{3}=z_{\mathrm{D}} x_{3}}^{\infty} f_{\boldsymbol{\gamma}^{\mathrm{sig}}}(\boldsymbol{y}) \mathrm{d} \boldsymbol{y} .
$$

The pdfs of $\gamma^{\text {sig }}$ for I.I.D., QSNR and QS channels are given by

$$
\begin{aligned}
& f_{\boldsymbol{\gamma}^{\text {sig }}}(\boldsymbol{y})=f_{\gamma_{\mathrm{R}}^{\text {sig }}}\left(y_{1}\right) f_{\gamma_{\mathrm{C}}^{\text {sig }}}\left(y_{2}\right) f_{\gamma_{\mathrm{D}}^{\text {sig }}}\left(y_{3}\right), \\
& f_{\boldsymbol{\gamma}^{\text {sig }}}(\boldsymbol{y})=f_{\gamma_{\mathrm{R}}^{\text {sig }}}\left(y_{1}\right) f_{\gamma_{\mathrm{C}}^{\text {sig }}}\left(y_{2}\right) \delta\left(y_{1}-y_{3}\right), \\
& f_{\boldsymbol{\gamma}^{\text {sig }}}(\boldsymbol{y})=f_{\gamma_{\mathrm{R}}^{\text {sig }}}\left(y_{1}\right) \delta\left(y_{1}-y_{2}\right) \delta\left(y_{1}-y_{3}\right),
\end{aligned}
$$

respectively. For Rayleigh-fading channels, (34) evaluates as

$$
\begin{gathered}
\mathcal{K}(\boldsymbol{x} ; \text { I.I.D. })=e^{-\eta_{1} / \bar{\gamma}}, \eta_{1}=z_{\mathrm{R}} x_{1}+z_{\mathrm{C}} x_{2}+z_{\mathrm{D}} x_{3}, \\
\mathcal{K}(\boldsymbol{x} ; \mathrm{QSNR})=e^{-\eta_{2} / \bar{\gamma}}, \eta_{2}=\max \left\{z_{\mathrm{R}} x_{1}, z_{\mathrm{D}} x_{3}\right\}+z_{\mathrm{C}} x_{2}, \\
\mathcal{K}(\boldsymbol{x} ; \mathrm{QS})=e^{-\eta_{3} / \bar{\gamma}}, \eta_{3}=\max \left\{z_{\mathrm{R}} x_{1}, z_{\mathrm{C}} x_{2}, z_{\mathrm{D}} x_{3}\right\} .
\end{gathered}
$$

Clearly $\eta_{1} \geq \eta_{2} \geq \eta_{3}$, hence $\mathcal{K}(\boldsymbol{x}$; I.I.D. $) \leq \mathcal{K}(\boldsymbol{x} ;$ QSNR $) \leq$ $\mathcal{K}(\boldsymbol{x} ; \mathrm{QS})$. It follows from (33) that the inequality (24) holds.

\section{APPENDIX IV}

DERIVATION OF (25)

For any generic channel type (GCT), which can be QS, QSNR or I.I.D., the DATA capture probability is given by

$$
\begin{aligned}
& \operatorname{Pr}\left(\mathcal{C}_{\mathrm{D}} ; \mathrm{GCT}\right) \\
= & \int \operatorname{Pr}\left(\mathcal{C}_{\mathrm{D}} \mid \mathbb{T}_{\text {cycle }} ; \mathrm{GCT}\right) f_{\mathbb{T}_{\text {cycle }}}\left(\mathbb{T}_{\text {cycle }} ; \mathrm{GCT}\right) \mathrm{d} \mathbb{T}_{\text {cycle }} .
\end{aligned}
$$

From (24) and (35), clearly (25) holds if the cycle traffic pdf does not depend on GCT, i.e., if

$$
f_{\mathbb{T}_{\text {cycle }}}\left(\mathbb{T}_{\text {cycle }} ; \text { GCT }\right)=f_{\mathbb{T}_{\text {cycle }}}\left(\mathbb{T}_{\text {cycle }}\right)
$$

regardless of GCT, for $\operatorname{Pr}\left(\mathcal{C}_{\mathrm{D}} \mid \mathbb{T}_{\text {cycle }} ; \mathrm{GCT}\right)>0$. To show that (36) indeed holds, we express the pdf of the cycle traffic as

$$
\begin{aligned}
f_{\mathbb{T}_{\text {cycle }}}\left(\mathbb{T}_{\text {cycle }} ; \mathrm{GCT}\right)= & f_{\mathbb{T}_{\mathrm{R}}}\left(\mathbb{T}_{\mathrm{R}} ; \mathrm{GCT}\right) f_{\mathbb{T}_{\mathrm{C}}}\left(\mathbb{T}_{\mathrm{C}} \mid \mathbb{T}_{\mathrm{R}} ; \mathrm{GCT}\right) \\
\times & f_{\mathbb{T}_{\mathrm{D}}}\left(\mathbb{T}_{\mathrm{D}} \mid \mathbb{T}_{\mathrm{R}}, \mathbb{T}_{\mathrm{C}} ; \mathrm{GCT}\right) .
\end{aligned}
$$

The DATA capture probability is positive only if past successive captures have occurred. Therefore, we can write $f_{\mathbb{T}_{\mathrm{C}}}\left(\mathbb{T}_{\mathrm{C}} \mid \mathbb{T}_{\mathrm{R}} ; \mathrm{GCT}\right)$ as $f_{\mathbb{T}_{\mathrm{C}}}\left(\mathbb{T}_{\mathrm{C}} \mid \mathbb{T}_{\mathrm{R}}, \mathcal{C}_{\mathrm{R}} ; \mathrm{GCT}\right)$ and $f_{\mathbb{T}_{\mathrm{D}}}\left(\mathbb{T}_{\mathrm{D}} \mid \mathbb{T}_{\mathrm{R}}, \mathbb{T}_{\mathrm{C}} ; \mathrm{GCT}\right) \quad$ as $\quad f_{\mathbb{T}_{\mathrm{D}}}\left(\mathbb{T}_{\mathrm{D}} \mid \mathbb{T}_{\mathrm{R}}, \mathbb{T}_{\mathrm{C}}, \mathcal{C}_{\mathrm{R}}, \mathcal{C}_{\mathrm{C}} ; \mathrm{GCT}\right)$. Regardless of GCT, knowing $\mathcal{C}_{\mathrm{R}}$ is sufficient to determine the pdf of $\mathbb{T}_{\mathrm{C}}$, and knowing $\mathcal{C}_{\mathrm{R}}$ and $\mathcal{C}_{\mathrm{C}}$ is sufficient to determine the pdf of $\mathbb{T}_{\mathrm{D}}$. Thus, (36) holds which completes the proof. 


\section{APPENDIX V \\ Obtaining Throughrut In QS Channels FOR $P \rightarrow \infty$}

We use a semi-analytical method to obtain the throughput $\overline{\bar{s}}\left(R_{\mathrm{D}}\right)$ in $\mathrm{QS}$ channels for $P \rightarrow \infty$, as follows. We note that $\overline{\mathcal{I}}_{\mathrm{D}}$ conditioned on a received signal power $\gamma\left(=\gamma_{\mathrm{R}}^{\text {sig }}=\gamma_{\mathrm{C}}^{\text {sig }}=\right.$ $\left.\gamma_{\mathrm{D}}^{\text {sig }}\right)$ approaches the constant $\widetilde{\mu}_{\mathcal{I}}(\gamma) \triangleq \mathbb{E}\left[\mathcal{I}_{\mathrm{D}} \mid \mathcal{C}_{\mathrm{R}}, \mathcal{C}_{\mathrm{C}}, \gamma\right]$ for large $P$. The asymptotic throughput is then given by $\overline{\bar{s}}\left(R_{\mathrm{D}}\right)=$ $R_{\mathrm{D}} \operatorname{Pr}\left(\widetilde{\mu}_{\mathcal{I}}(\gamma)>R_{\mathrm{D}} \mid \mathcal{C}_{\mathrm{R}}, \mathcal{C}_{\mathrm{C}}\right)$, where $\gamma$ is taken as the random variable. Hence, by obtaining samples of $\widetilde{\mu}_{\mathcal{I}}(\gamma)$ from Monte Carlo simulations, $\overline{\bar{s}}\left(R_{\mathrm{D}}\right)$ can be computed numerically.

\section{ACKNOWLEDGMENT}

The authors gratefully acknowledge the constructive remarks of the anonymous reviewers which significantly improve the quality of the paper. The authors also thank Jan Bergmans for his valuable comments, and Xiangyu Wang and Dee Denteneer for helpful discussions.

\section{REFERENCES}

[1] IEEE J. Sel. Areas Commun., special issue on wireless ad hoc networks, vol. 17, Aug. 1999.

[2] N. Abramson, "The ALOHA system-another alternative for computer communications," in Proc. Fall Joint Computer Conf., AFIPS Conf., vol. 37, 1970.

[3] L. Kleinrock and F. Tobagi, "Packet switching in radio channelspart I: carrier sense multiple-access modes and their throughput-delay characteristics," IEEE Trans. Commun., vol. 23, no. 12, pp. 1400-1416, Dec. 1975.

[4] P. Karn, "MACA-a new channel access method for packet radio," in Proc. ARRL/CRRL Amateur Radio 9th Computer Networking Conference, Sept. 1990, pp. 134-140.

[5] V. Bharghavan, A. Demers, S. Shenker, and L. Zhang, "MACAW: a media access protocol for wireless LAN's," in Proc. SIGCOMM'94, Conf. on Communications Architectures, Protocols and Applications, Aug. 1994, pp. 212-225.

[6] C. van der Plas and J. P. M. G. Linnartz, "Stability of mobile slotted aloha network with Rayleigh fading, shadowing, and near-far effect," IEEE Trans. Veh. Technol., vol. 39, no. 4, pp. 359-366, Nov. 1990.

[7] Part 11: Wireless LAN Medium Access Control (MAC) and Physical Layer (PHY) Specifications, IEEE 802.11 Standard, 1999.

[8] S. Ray, J. B. Carruthers, and D. Starobinski, "RTS/CTS-induced congestion in ad hoc wireless LANs," in Proc. IEEE WCNC 2003, Mar. 2003, pp. 1516-1521.

[9] K. Xu, M. Gerla, and S. Bae, "Effectiveness of RTS/CTS handshake in IEEE 802.11 based ad hoc networks," Ad Hoc Networks, vol. 1, no. 1, pp. 107-123, July 2003.

[10] G. Bianchi, "IEEE 802.11-saturation throughput analysis," IEEE Commun. Lett., vol. 2, no. 12, pp. 318-320, Dec. 1998.

[11] N. Abramson, "The throughput of packet broadcasting channels," IEEE Trans. Commun., vol. 25, no. 1, pp. 117-128, Jan. 1977.

[12] J. Arnbak and W. van Blitterswijk, "Capacity of slotted ALOHA in Rayleigh-fading channels," IEEE J. Sel. Areas Commun., vol. 5, no. 2, pp. 261-269, Feb. 1987.

[13] E. S. Sousa, "Performance of a spread spectrum packet radio network link in a Poisson field of interferers," IEEE Trans. Inf. Theory, vol. 38, no. 6, pp. 1743-1754, Nov. 1992.

[14] J. P. M. G. Linnartz, R. Hekmat, and R.-J. Venema, "Near-far effects in land mobile random access networks with narrow-band Rayleigh fading channels," IEEE Trans. Veh. Technol., vol. 41, no. 1, pp. 77-90, Feb. 1992.

[15] J. P. M. G. Linnartz, "Slotted ALOHA land-mobile radio networks with site diversity," IEE Proc.-I, vol. 139, no. 1, pp. 58-70, Feb. 1992.
[16] Z. Hadzi-Velkov and B. Spasenovski, "Capture effect in IEEE 802.11 basic service area under influence of Rayleigh fading and near/far effect," in Proc. 13th IEEE Personal, Indoor and Mobile Radio Communications, vol. 1, Sept. 2002, pp. 172-176.

[17] J. H. Kim and J. K. Lee, "Capture effects of wireless CSMA/CA protocols in Rayleigh and shadow fading channels," IEEE Trans. Veh. Technol., vol. 48, no. 4, pp. 1277-1286, July 1999.

[18] R. Knopp and P. A. Humblet, "On coding for block fading channels," IEEE Trans. Inf. Theory, vol. 46, no. 1, pp. 189-205, Jan. 2000.

[19] L. H. Ozarow, S. Shamai, and A. D. Wyner, "Information theoretic considerations for cellular mobile radio," IEEE Trans. Veh. Technol. vol. 43, no. 2, pp. 359-378, May 1994.

[20] G. Caire and D. Tuninetti, "The throughput of hybrid-ARQ protocols for the Gaussian collision channel," IEEE Trans. Inf. Theory, vol. 47, no. 5, pp. 1971-1988, July 2001.

[21] Part 11: Wireless LAN Medium Access Control (MAC) and Physical Layer (PHY) Specifications - Amendment 8: Medium Access Control (MAC) Quality of Service Enhancements Enhancements, IEEE 802.11e Standard, Nov. 2005.

[22] R. G. Gallager, Discrete Stochastic Processes. Boston: Kluwer Academic Publishers, 1996

[23] P. A. W. Lewis and G. S. Shedler, "Simulation of nonhomogeneous Poisson processes by thinning," Naval Res. Logistics Quart, vol. 26, pp. 403-413, 1979.

[24] C. K. Ho and J. P. M. G. Linnartz, "Analysis of the RTS/CTS multiple access scheme with capture effect," in Proc. 17th IEEE Personal, Indoor and Mobile Radio Communications, Sept. 2006, pp. 1-5.

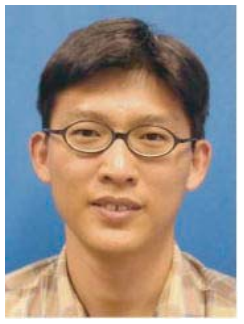

Chin Keong Ho received the B.Eng. (first-class Honors) and M. Eng degrees from the Department of Electrical Engineering, National University of Singapore in 1999 and 2001, respectively. Since 2001, he is with the Institute for Infocomm Research, Singapore. He took leave in 2004-2007 to work toward the Ph.D. degree at Eindhoven University of Technology, The Netherlands, during which he conducted joint research with Philips Research Laboratories, Eindhoven, The Netherlands. His research interest lies in adaptive wireless communications and signal processing for multicarrier and space-time communications.

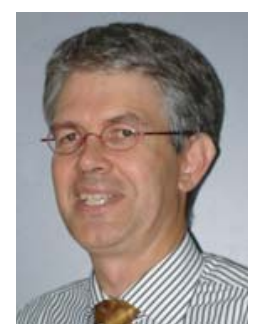

Jean-Paul Linnartz received his M.Sc. degree Cum Laude from Eindhoven University of Technology, The Netherlands, in 1986. During 1987-1988, he worked with the Physics and Electronics Laboratory (F.E.L-T.N.O., The Hague) of the Netherlands Organization for Applied Scientific Research in the field of frequency planning and UHF propagation. From 1988-1991, he was Assistant Professor at Delft University of Technology, where in 1991 he received his $\mathrm{Ph}$.D. Cum Laude on multi-user mobile radio nets. In 1992-1995, he was an Assistant Professor at The University of California at Berkeley. He introduced the concept and the name Multi-Carrier CDMA. In 1994, he became Associate Professor at Delft University of Technology in The Netherlands. In 1995, he joined Royal Philips Electronics to set up a research activity on content protection, security and digital rights management (DRM) at Philips Research, Eindhoven, The Netherlands. As a Principal Scientist, he actively participated in various standardization forums. He applied detection-theoretical concepts to improve the detection of electronic watermarks, and invented privacypreserving authentication schemes based on biometrics. In 2001 he became a Department Head. His group developed for instance content protection schemes for optical disc standards, including Super-Audio CD, and a video watermarking system which is in commercial use to monitor television content worldwide. Since 2003, he is Senior Director of the Connectivity Systems and Networks (CoSiNe) department, which plays an active role for instance in the 802.11 standardization for wireless LANs. He and his group realized a Doppler compensation scheme for mobile reception of DVB-T and DVB-H. Since 2006, he is part-time professor at Eindhoven University of Technology. $\mathrm{He}$ has 25 issued patents, mainly in the field of electronic watermarking and communications. 